\title{
Association Analysis and Estimation of Breeding Values of Iranian Table Grapevine Germplasm by Integrating Pomological and Molecular Data
}

Mitra Razi

Urmia University

Reza Darvishzadeh ( $\nabla$ r.darvishzadeh@urmia.ac.ir)

Urmia University

Hamed Baneh

Agricultural and Natural Resources Research and Education Center

Hadi Alipour

Urmia University

\section{Research Article}

Keywords: Genetic resources, breeding values, Iranian, grapevine germplasm, pomological and molecular data, Table grape

Posted Date: September 30th, 2021

DOI: https://doi.org/10.21203/rs.3.rs-936436/v1

License: () (1) This work is licensed under a Creative Commons Attribution 4.0 International License. Read Full License 


\section{Abstract}

Genetic resources in each country are valuable assets for sustainable development. An accurate knowledge of genetic behavior and identification of genomic loci associated with important economic traits will help breeders to run efficiently their breeding programs. Fourteen important pomological traits were measured on 45 Iranian table grape cultivars during three successive years. Molecular profile of studied cultivars was prepared with 39 (Simple sequence Repeat) SSR primers pairs. Genetic structure analysis based on the SSR markers revealed two sub-populations $(\mathrm{K}=2)$ in the association panel. In association analysis using mixed linear model seven loci were found to be significantly associated with the studied traits at $5 \%$ probability level. Breeding values were also estimated for the pomological traits using the best linear unbiased prediction (BLUP). "Saghal Solian”, "At Ouzum", "Garmian”, "Rishbaba Qermez", "Taifi”, "Shahroudi”, "Sahebi Qermez”, “Lal Qermez”, "Alhaghi”, “Sarghola”, “Chava Ga”, "Qzl Ouzum” and "Agh Shani” table grape cultivars showed high and positive breeding value for cluster length, width and weight. Whereas "Garmian", "Rishbaba Qermez", "Fakhri", "Agh Shani”, "Lal Sefid" and "Shirazi" had positive and high breeding value for pollen germination, fruit set in open pollination and fruit set under controlled pollination. Finally, Sarghola" and "Qzl Ouzum" showed the positive and highest breeding value for berry weight, flesh weight, cluster length, cluster width and cluster weight. The cultivar with high and positive breeding value can be used as a good parent for breeding of traits in hybridization programs because they can better transfer the desirable characteristics to the progeny in each case.

\section{Introduction}

Table grape (Vitis vinifera L.) is one of the most important horticultural crops and it is a human interest in many centuries ${ }^{12}$. Archaeological studies have shown that Iran is one of the centers of domestication of grape ${ }^{41}$. The size of the grape genome with 38 chromosomes is about $4.75 \times 108$ bp. This genome is a combination of duplicate and non-duplicate sequences that duplicate DNA makes up more than $95 \%$ of the genome ${ }^{26}$. Investigation of genetic basis of quantitative traits has become possible by the development of molecular markers ${ }^{58,50}$. In many plants, identification of genomic regions controlling quantitative traits could provide valuable genetic information about the genetic basis and structure of complex traits ${ }^{25}$. The development of molecular marker technology together with appropriate statistical genomic techniques has facilitated the achievement of this goal. Identification of the genomic regions associated with quantitative inheritance is usually accomplished by two main methods: linkage mapping and association analysis. Recently the use of linkage mapping has been limited due to numerous limitations. For instance, production of artificial populations as a prerequisite is difficult especially in the fruit trees ${ }^{27}$

For overcome linkage mapping limitations, association analysis has been widely used in mapping programs in the recent years. In this method, the relationship between genotype and phenotype of individuals coming from natural populations is examined directly to identify the chromosomal regions involved in controlling trait variations ${ }^{48}$. Unlike linkage analysis in which the linkage disequilibrium is only due to physical linkage, in natural populations used in association analysis the linkage disequilibrium is caused in addition to physical linkage by other factors such as small population size, migration and so on. Recent factors, however, led to the identification of false positive markers that are not important from the point of plant breeding view ${ }^{70}$. So, in order to decrease false positive associations, it is necessary to consider population structure and kinship in association models ${ }^{68}$.

On the other hand, several studies were conducted in grape via genome wide association analysis to identify genomic regions involved in important traits such as berry skin ${ }^{24,37}$, berry proanthocyanidin compound ${ }^{34}$ and fleshless berry ${ }^{33}$. QTLs effective on fruit yield in grape using AFLP (Amplified Fragment Length Polymorphism) and SSR (Simple Sequence Repeat) markers were identified ${ }^{19}$. Also, in grapevine some association studies have been conducted via candidate genes approach including $\mathrm{Myb}^{57,23}, \mathrm{VvDXS}^{16}, \mathrm{VvPel}^{61}, \mathrm{VvGAl}^{60}, \mathrm{VvTFL} 1 \mathrm{~A}^{21}$ and 183 candidate genes associated with cluster architecture ${ }^{56}$. In a study, genetic basis of grapevine leaf shape was reported for the first time by genome wide association analysis (GWAS) including 961 grapevine accessions genotyped with 6114 SNPs $^{11}$.

Selecting parents for hybridization programs is always a great challenge in front of plant breeders. Several methodologies have been utilized in helping the identification of genotypes with promising and appropriate agronomical characters for hybridization. Parent selection based on the predicted breeding values $^{29,28,65}$ is a method extensively used in animal breeding for a long time ${ }^{18}$. Two common methods for predicting the breeding value of parents are selection index and BLUP. In the selection index, it is not possible to estimate the effect of environmental factors, so the data used in this method must be corrected for environmental factors before using. In fact, by this method the best predict of breeding value is estimated in populations with same environment conditions. For this reason, in recent years this method is less used for predicting breeding value. On the contrary, in the BLUP method, corrected data for environmental factors is not required for statistical analysis. Another advantage of this method is the use of all kinship relationships in predicting of breeding value. Genetic change that is occurred in populations with continuous genetic selection, can be estimated by BLUP.

Another feature of this method is its using for multiple traits ${ }^{5}$. The type of the model used depends on the specificity of the data and the purpose of breeding programs. When the phenotypic data come from different conditions, they should be tested using robust statistical procedures for correcting the data for disturbing factors in order to increase the heritability coefficient, which will increase the accuracy of selection. Traditionally, for estimating breeding values (BLUP), pedigree information of lines is used in software package such as Wombat ${ }^{40}$. It is possible to use Kinship matrix instead of genetic relationship matrix (pedigree information) for predicting breeding values in the model ${ }^{4}$. In BLUP context, the main purpose of breeders is to increase the correlation of true genotypic values and predicted genotypic values that the method can maximize this correlation ${ }^{49}$.

The objectives of the present study are to find SSR loci associated with pomological traits using association analysis and to evaluate the breeding values for selecting the best cultivars from Iranian table grape germplasm by integrating the pomological and molecular data. The outputs of the present study are introducing appropriate genotypes as parents based on breeding values for constructing source populations and associated SSR markers that can be used for 
selecting desirable individuals from the source populations during grape breeding programs. This has an advantage to progeny test. The major disadvantage of progeny test in fruit trees is their long generation and also necessarily having wide areas for plant evaluation.

\section{Results}

Descriptive phenotypic analysis. High levels of phenotypic variation were observed among the studied table grape cultivars for studied pomological traits. The highest mean value based on three years' data was observed in cluster weight (544.95). The highest broad sense heritability (98.46\%) was observed in pollen germination rate (Table 1). A negative correlation was observed between fruit pH and TA. A positive correlation was observed between berry weight and flesh weight, as well as between fruit set under controlled pollination and pollen germination (Table 2). In cluster analysis based on agro-morphological characters, the studied genotypes were sub-divided into four main groups (Fig. 1).

Table 1

Descriptive statistics for agronomic traits of grape cultivars during three successive years

\begin{tabular}{|c|c|c|c|c|c|c|c|c|c|c|c|}
\hline \multirow[t]{2}{*}{ Trait } & \multicolumn{4}{|l|}{ Year 1} & \multicolumn{3}{|l|}{ Year 2} & \multicolumn{4}{|l|}{ Year 3} \\
\hline & Range & Mean & \multicolumn{2}{|c|}{ Std. Deviation } & Range & Mean & Deviation & \multicolumn{2}{|c|}{ Range } & Mean & $\begin{array}{l}\text { Std. } \\
\text { Deviation }\end{array}$ \\
\hline TSS & $14.8-24.6$ & 18.95 & \multicolumn{2}{|c|}{2.64} & $14.5-26$ & 19.86 & 3.06 & \multicolumn{2}{|c|}{$15-26.5$} & 18.86 & 2.49 \\
\hline $\mathrm{Ph}$ & $2.22-5.4$ & 3.04 & \multicolumn{2}{|c|}{0.68} & $3.1-5.4$ & 3.56 & 0.34 & \multicolumn{2}{|c|}{$2.56-4.76$} & 3.38 & 0.34 \\
\hline TA & $0.09-2.6$ & 0.8 & \multicolumn{2}{|c|}{0.51} & $\begin{array}{l}0.09- \\
1.51\end{array}$ & 0.59 & 0.23 & \multicolumn{2}{|c|}{$0.09-1.12$} & 0.65 & 0.22 \\
\hline BW & $1.31-5.15$ & 3.02 & \multicolumn{2}{|c|}{1.08} & $\begin{array}{l}1.36- \\
5.15\end{array}$ & 3.01 & 1.05 & \multicolumn{2}{|c|}{$1.44-6.5$} & 3.44 & 1.28 \\
\hline FW & $1.23-4.93$ & 2.88 & \multicolumn{2}{|c|}{1.05} & $\begin{array}{l}1.36- \\
5.05\end{array}$ & 2.91 & 1.02 & \multicolumn{2}{|c|}{$1.43-6.4$} & 3.36 & 1.26 \\
\hline SSW & $0-0.15$ & 0.06 & \multicolumn{2}{|c|}{0.03} & $0-0.15$ & 0.05 & 0.03 & \multicolumn{2}{|l|}{$0-0.15$} & 0.03 & 0.02 \\
\hline SN & $0.95-3.5$ & 2.13 & \multicolumn{2}{|c|}{0.64} & $1.1-3.4$ & 2.09 & 0.62 & $1-3.5$ & & 2.2 & 0.61 \\
\hline JV & $31-49$ & 41.32 & & & $30-50$ & 42.13 & 4.22 & $32-51$. & & 43.77 & 4.73 \\
\hline FSOP & $10.6-63.3$ & 32.6 & & & $10-76$ & 31.07 & 13.42 & $5.6-64$ & & 32.42 & 11.46 \\
\hline$P G$ & $0.5-65.5$ & 28.3 & & & $1.2-65$ & 28.95 & 17.15 & $0.4-62$ & & 26.07 & 16.16 \\
\hline $\mathrm{CL}$ & $9-26$ & 18.83 & & & $9-32.7$ & 19.68 & 4.65 & $13-26$ & & 19.91 & 3.27 \\
\hline CWid & $5-17.5$ & 10.1 & & & $4-20$ & 10.01 & 3.18 & $5.5-15$ & & 10.13 & 2.25 \\
\hline CW & $\begin{array}{l}67.1- \\
874.9\end{array}$ & 402.37 & & & $300-950$ & 636 & 132.21 & 223.3- & 387 & 597.61 & 268.52 \\
\hline FSCP & $0-58.5$ & 24.65 & & & $0-70.9$ & 27.32 & 16.18 & $1.8-51$ & & 24.34 & 11.99 \\
\hline Trait & Thr & e years $\mathrm{c}$ & & & & PCV & GCV & $h^{2} b s$ & GA5\% & & GA5\% mean \\
\hline & Ran & & Mean & Std. De & & & & & & & \\
\hline TSS & 15.3 & 3-23.56 & 19.22 & 2.17 & & 11.35 & 9.58 & 71.23 & 3.20 & & 16.66 \\
\hline $\mathrm{Ph}$ & 2.8 & -5.03 & 3.32 & 0.38 & & 11.41 & 9.47 & 68.83 & 0.54 & & 16.18 \\
\hline TA & 0.05 & -1.38 & 0.68 & 0.24 & & 35.46 & 24.22 & 46.65 & 0.23 & & 34.08 \\
\hline BW & $1.4-$ & & 3.14 & 1.05 & & 33.31 & 31.82 & 91.25 & 1.98 & & 62.62 \\
\hline FW & 1.35 & -4.92 & 3.04 & 1.03 & & 33.65 & 32.05 & 90.75 & 1.92 & & 62.90 \\
\hline SSW & $0-0$. & & 0.05 & 0.02 & & 50.14 & 44.12 & 77.43 & 0.04 & & 79.98 \\
\hline SN & 1.03 & -3.2 & 2.13 & 0.55 & & 25.70 & 23.87 & 86.25 & 0.98 & & 45.67 \\
\hline JV & 20.7 & -50.26 & 41.96 & 5.19 & & 9.60 & 9.41 & 96.25 & 8.07 & & 19.03 \\
\hline FSOP & 8.7- & 7.7 & 31.98 & 11.02 & & 34.43 & 32.48 & 88.95 & 20.21 & & 63.09 \\
\hline PG & $1-6$ & & 27.27 & 16.97 & & 59.53 & 59.07 & 98.46 & 33.53 & & 120.74 \\
\hline $\mathrm{CL}$ & 12.2 & -24.7 & 19.44 & 3.24 & & 16.59 & 13.51 & 66.32 & 4.41 & & 22.66 \\
\hline CWid & $5-15$ & & 10.24 & 2.38 & & 22.01 & 18.65 & 71.77 & 3.28 & & 32.55 \\
\hline $\mathrm{CW}$ & 326 & 905 & 544.95 & 145.92 & & 26.75 & 19.46 & 52.90 & 158.96 & & 29.15 \\
\hline FSCP & 3.8 & & 25.4 & 10.79 & & 42.54 & 35.00 & 67.69 & 15.09 & & 59.32 \\
\hline
\end{tabular}


PCV: Phenotypic coefficient of variation, GCV: Genotypic coefficient of variation, $h^{2}$ bs: Narrow sense heritability, GA $5 \%$ : Genetic advance in 5 percent probability level. TSS: Total soluble solids, TA: Titratable acidity, BW: Berry weight, FW: Flesh weight, SSW: Single seed weight, SN: Seed number, JV: Juice volume, FSOP: Fruit set in open pollination, PG: Pollen germination, CL: Cluster length, Cwi: Cluster width, CW: Cluster weight, FSCP: Fruit set under controlled pollination.

Table 2

Correlation coefficients among agronomic traits of grape cultivars in the first (upper diagonal) and second (low diagonal) cropping season

\begin{tabular}{|c|c|c|c|c|c|c|c|c|c|c|c|c|c|}
\hline & TSS & $\mathrm{Ph}$ & TA & BW & FW & SSW & SN & JV & FSOP & PG & $\mathrm{CL}$ & CWid & CW \\
\hline TSS & 1 & $0.196^{\mathrm{ns}}$ & $-0.105^{\mathrm{ns}}$ & $-0.296 *$ & $-0.293^{\mathrm{ns}}$ & $\begin{array}{l}-0.210 \\
\mathrm{~ns}\end{array}$ & $-0.011^{n s}$ & $-0.051^{\mathrm{ns}}$ & $0.188^{n s}$ & $0.328^{*}$ & $0.069 \mathrm{~ns}$ & $0.110^{n s}$ & $0.192^{r}$ \\
\hline $\mathrm{Ph}$ & $0.209^{n s}$ & 1 & $-0.494^{\star \star}$ & $0.194^{\mathrm{ns}}$ & $0.206^{n s}$ & $\begin{array}{l}-0.049 \\
\mathrm{~ns}\end{array}$ & $0.10493^{\mathrm{ns}}$ & $-0.252^{\mathrm{ns}}$ & $0.003^{n s}$ & $0.112^{\mathrm{ns}}$ & $\begin{array}{l}-0.105 \\
\mathrm{~ns}\end{array}$ & $-0.258^{n s}$ & $0.166^{r}$ \\
\hline TA & $-0.175^{\mathrm{ns}}$ & $-0.621^{\star \star}$ & 1 & $-0.271^{\mathrm{ns}}$ & $-0.275^{\mathrm{ns}}$ & $\begin{array}{l}-0.012 \\
\mathrm{~ns}\end{array}$ & $-0.096^{n s}$ & $0.123^{n s}$ & $0.264^{\mathrm{ns}}$ & $-0.000 \mathrm{~ns}$ & $0.201^{n s}$ & $0.205^{\mathrm{ns}}$ & $\begin{array}{l}-0.015 \\
\mathrm{~ns}\end{array}$ \\
\hline BW & $\begin{array}{l}-0.242 \\
n s\end{array}$ & $0.105^{\mathrm{ns}}$ & $-0.170^{\text {ns }}$ & 1 & $0.997 * \star$ & $0.388^{\star *}$ & $0.315^{*}$ & $0.119^{\mathrm{ns}}$ & $-0.071^{\mathrm{ns}}$ & $-0.274^{n s}$ & $\begin{array}{l}-0.047 \\
n s\end{array}$ & $0.193^{\mathrm{ns}}$ & 0.330 * \\
\hline FW & $-0.248^{n s}$ & $0.111^{\mathrm{ns}}$ & $-0.164^{\mathrm{ns}}$ & $0.998 * \star$ & 1 & $0.327^{*}$ & $0.283^{n s}$ & $0.108^{n s}$ & $-0.077^{\mathrm{ns}}$ & $-0.264^{n s}$ & $\begin{array}{l}-0.061 \\
\mathrm{~ns}\end{array}$ & $0.175^{\mathrm{ns}}$ & $0.324^{*}$ \\
\hline SSW & $\begin{array}{l}-0.181 \\
\mathrm{~ns}\end{array}$ & $-0.128^{n s}$ & $0.001^{n s}$ & $0.375^{\star}$ & $0.332^{\star}$ & 1 & $0.063^{n s}$ & $0.133^{n s}$ & $-0.141^{\mathrm{ns}}$ & $-0.296^{\star}$ & $0.074^{n s}$ & $0.091^{n s}$ & $\begin{array}{l}0.075 \\
\mathrm{~ns}\end{array}$ \\
\hline SN & $0.227^{\mathrm{ns}}$ & $0.038^{n s}$ & $-0.177^{n s}$ & $0.185^{n s}$ & $0.171^{\mathrm{ns}}$ & $-0.235^{\mathrm{ns}}$ & 1 & $0.241^{\mathrm{ns}}$ & $0.307^{*}$ & $0.112^{\mathrm{ns}}$ & $0.056^{n s}$ & $0.369 *$ & $0.308^{*}$ \\
\hline JV & $\begin{array}{l}-0.144 \\
\mathrm{~ns}\end{array}$ & $-0.092^{n s}$ & $-0.073^{n s}$ & $0.185^{n s}$ & $0.185^{\mathrm{ns}}$ & $-0.035^{\mathrm{ns}}$ & $0.228^{\mathrm{ns}}$ & 1 & $0.136^{\mathrm{ns}}$ & $0.048^{n s}$ & $0.203^{n s}$ & $0.313^{*}$ & $0.440 *$ \\
\hline FSOP & $0.294^{\star}$ & $0.325^{\star}$ & $-0.296^{\mathrm{ns}}$ & $-0.072^{\mathrm{ns}}$ & $-0.072^{\mathrm{ns}}$ & $-0.175^{\mathrm{ns}}$ & $0.216^{\mathrm{ns}}$ & $0.012^{n s}$ & 1 & $0.2917^{\mathrm{ns}}$ & $\begin{array}{l}-0.129 \\
\mathrm{~ns}\end{array}$ & $0.259 \mathrm{~ns}$ & $\begin{array}{l}0.142 \\
\mathrm{~ns}\end{array}$ \\
\hline PG & $0.129 \mathrm{~ns}$ & $0.102^{\mathrm{ns}}$ & $-0.148^{\text {ns }}$ & $-0.338^{*}$ & $-0.323^{\star}$ & $-0.503^{\star \star}$ & $0.274^{\mathrm{ns}}$ & $0.008^{n s}$ & $0.148^{n s}$ & 1 & $\begin{array}{l}-0.235 \\
\mathrm{~ns}\end{array}$ & $0.255^{\mathrm{ns}}$ & $\begin{array}{l}0.076 \\
\mathrm{~ns}\end{array}$ \\
\hline $\mathrm{CL}$ & $0.045^{n s}$ & $0.244^{\mathrm{ns}}$ & $-0.066^{n s}$ & $0.157^{n s}$ & $0.164^{\mathrm{ns}}$ & $\begin{array}{l}-0.141 \\
\mathrm{~ns}\end{array}$ & $0.106^{\mathrm{ns}}$ & $0.176^{\mathrm{ns}}$ & $0.053^{n s}$ & $-0.090^{\mathrm{ns}}$ & 1 & $0.206^{\mathrm{ns}}$ & $\begin{array}{l}0.177 \\
\mathrm{~ns}\end{array}$ \\
\hline CWid & $0.018^{n s}$ & $0.115^{\mathrm{ns}}$ & $-0.235^{\mathrm{ns}}$ & $0.157^{n s}$ & $0.164^{\mathrm{ns}}$ & $\begin{array}{l}-0.234 \\
\mathrm{~ns}\end{array}$ & $0.292^{\mathrm{ns}}$ & $0.447 \star \star$ & $0.390 * \star$ & $0.030^{\mathrm{ns}}$ & $0.372^{\star}$ & 1 & $0.563^{*}$ \\
\hline CW & $\begin{array}{l}-0.207 \\
n s\end{array}$ & $-0.198^{\mathrm{ns}}$ & $0.002^{n s}$ & $0.499 \star \star$ & $0.497^{\star \star}$ & $0.172^{\mathrm{ns}}$ & $0.203^{\mathrm{ns}}$ & $0.417 \star \star$ & $0.139^{\mathrm{ns}}$ & $-0.173^{\mathrm{ns}}$ & $0.141^{\mathrm{ns}}$ & $0.364^{*}$ & 1 \\
\hline FSCP & $0.071^{\mathrm{ns}}$ & $0.225^{n s}$ & $-0.276^{\mathrm{ns}}$ & $0.073^{n s}$ & $0.077^{n s}$ & $\begin{array}{l}-0.190 \\
\mathrm{~ns}\end{array}$ & $0.290^{\mathrm{ns}}$ & $-0.182^{\mathrm{ns}}$ & $0.473^{\star \star}$ & $0.168^{\mathrm{ns}}$ & $-0.034^{\mathrm{ns}}$ & $0.193^{n s}$ & $\begin{array}{l}0.061 \\
\mathrm{~ns}\end{array}$ \\
\hline
\end{tabular}


Table 2

(Continuation). Correlation coefficients among agronomic traits of grape cultivars in the third (upper diagonal) and mean of three (low diagonal) cropping sea

\begin{tabular}{|c|c|c|c|c|c|c|c|c|c|c|c|c|c|c|}
\hline & TSS & $\mathrm{Ph}$ & TA & BW & FW & SSW & SN & JV & FSOP & PG & $\mathrm{CL}$ & CWid & $\mathrm{CW}$ & FSC \\
\hline TSS & 1 & $0.041^{\mathrm{ns}}$ & $0.157^{\mathrm{ns}}$ & $\begin{array}{l}-0.176 \\
\text { ns }\end{array}$ & $\begin{array}{l}-0.165 \\
\text { ns }\end{array}$ & $\begin{array}{l}-0.275 \\
\mathrm{~ns}\end{array}$ & $\begin{array}{l}0.026 \\
\mathrm{~ns}\end{array}$ & $\begin{array}{l}0.077 \\
\mathrm{~ns}\end{array}$ & $\begin{array}{l}0.138 \\
\mathrm{~ns}\end{array}$ & $0.350^{*}$ & $\begin{array}{l}0.003 \\
\mathrm{~ns}\end{array}$ & $\begin{array}{l}-0.011 \\
\text { ns }\end{array}$ & $\begin{array}{l}0.014 \\
\mathrm{~ns}\end{array}$ & $\begin{array}{l}0.2 \text { ? } \\
\mathrm{ns}\end{array}$ \\
\hline $\mathrm{Ph}$ & $\begin{array}{l}0.149 \\
\mathrm{~ns}\end{array}$ & 1 & $-0.519 * \star$ & $\begin{array}{l}-0.205 \\
\text { ns }\end{array}$ & $\begin{array}{l}-0.210 \\
\mathrm{~ns}\end{array}$ & $\begin{array}{l}0.159 \\
\mathrm{~ns}\end{array}$ & $\begin{array}{l}-0.146 \\
\text { ns }\end{array}$ & $\begin{array}{l}-0.176 \\
\mathrm{~ns}\end{array}$ & $\begin{array}{l}0.014 \\
\mathrm{~ns}\end{array}$ & $0.210^{\mathrm{ns}}$ & $\begin{array}{l}-0.082 \\
\text { ns }\end{array}$ & $\begin{array}{l}0.056 \\
\mathrm{~ns}\end{array}$ & $\begin{array}{l}0.013 \\
\mathrm{~ns}\end{array}$ & $\begin{array}{l}0.0 \text { ? } \\
\mathrm{ns}\end{array}$ \\
\hline TA & $\begin{array}{l}0.085 \\
\mathrm{~ns}\end{array}$ & $-0.664^{\star \star}$ & 1 & $\begin{array}{l}-0.047 \\
\text { ns }\end{array}$ & $\begin{array}{l}-0.047 \\
\text { ns }\end{array}$ & $\begin{array}{l}-0.062 \\
n s\end{array}$ & $\begin{array}{l}-0.152 \\
\text { ns }\end{array}$ & $\begin{array}{l}-0.014 \\
\text { ns }\end{array}$ & $\begin{array}{l}-0.068 \\
\text { ns }\end{array}$ & $\begin{array}{l}-0.235 \\
\mathrm{~ns}\end{array}$ & $\begin{array}{l}-0.016 \\
\text { ns }\end{array}$ & $\begin{array}{l}-0.242 \\
\mathrm{~ns}\end{array}$ & $\begin{array}{l}-0.039 \\
\text { ns }\end{array}$ & $\begin{array}{l}-0.1 \\
\mathrm{~ns}\end{array}$ \\
\hline BW & $-0.339 *$ & $\begin{array}{l}-0.004 \\
\mathrm{~ns}\end{array}$ & $\begin{array}{l}-0.259 \\
\text { ns }\end{array}$ & 1 & $0.999 * *$ & $\begin{array}{l}0.147 \\
\mathrm{~ns}\end{array}$ & $\begin{array}{l}0.239 \\
\mathrm{~ns}\end{array}$ & $\begin{array}{l}0.083 \\
\mathrm{~ns}\end{array}$ & $\begin{array}{l}-0.095 \\
\text { ns }\end{array}$ & $-0.453^{\star *}$ & $\begin{array}{l}0.085 \\
\mathrm{~ns}\end{array}$ & $\begin{array}{l}-0.156 \\
\text { ns }\end{array}$ & $\begin{array}{l}0.071 \\
\mathrm{~ns}\end{array}$ & $\begin{array}{l}-0.0 \\
\text { ns }\end{array}$ \\
\hline FW & -0.336 * & $\begin{array}{l}-0.006 \\
\mathrm{~ns}\end{array}$ & $\begin{array}{l}-0.258 \\
\text { ns }\end{array}$ & $0.999 * *$ & 1 & $\begin{array}{l}0.109 \\
\text { ns }\end{array}$ & $\begin{array}{l}0.224 \\
\mathrm{~ns}\end{array}$ & $\begin{array}{l}0.081 \\
\mathrm{~ns}\end{array}$ & $\begin{array}{l}-0.107 \\
\text { ns }\end{array}$ & $-0.454^{\star \star}$ & $\begin{array}{l}0.095 \\
\text { ns }\end{array}$ & $\begin{array}{l}-0.169 \\
\text { ns }\end{array}$ & $\begin{array}{l}0.071 \\
\mathrm{~ns}\end{array}$ & $\begin{array}{l}-0.0 \\
\text { ns }\end{array}$ \\
\hline SSW & -0.300 * & $0.006^{n s}$ & $\begin{array}{l}-0.046 \\
\text { ns }\end{array}$ & 0.420 ** & $0.383^{\star \star}$ & 1 & $\begin{array}{l}-0.047 \\
\mathrm{~ns}\end{array}$ & $\begin{array}{l}0.052 \\
\mathrm{~ns}\end{array}$ & $\begin{array}{l}0.165 \\
\mathrm{~ns}\end{array}$ & $\begin{array}{l}-0.001 \\
\mathrm{~ns}\end{array}$ & $\begin{array}{l}-0.209 \\
\text { ns }\end{array}$ & $\begin{array}{l}0.143 \\
\mathrm{~ns}\end{array}$ & $\begin{array}{l}-0.090 \\
\text { ns }\end{array}$ & $\begin{array}{l}0.06 \\
\mathrm{~ns}\end{array}$ \\
\hline $\mathrm{SN}$ & $\begin{array}{l}0.115 \\
\mathrm{~ns}\end{array}$ & $0.033^{\mathrm{ns}}$ & $\begin{array}{l}-0.238 \\
\text { ns }\end{array}$ & $\begin{array}{l}0.234 \\
\mathrm{~ns}\end{array}$ & $\begin{array}{l}0.218 \\
\mathrm{~ns}\end{array}$ & $\begin{array}{l}-0.113 \\
\mathrm{~ns}\end{array}$ & 1 & $\begin{array}{l}0.149 \\
\mathrm{~ns}\end{array}$ & $\begin{array}{l}0.455 \\
\mathrm{~ns}\end{array}$ & $0.071^{\mathrm{ns}}$ & $\begin{array}{l}-0.073 \\
\text { ns }\end{array}$ & $\begin{array}{l}0.255 \\
\mathrm{~ns}\end{array}$ & $\begin{array}{l}0.187 \\
\mathrm{~ns}\end{array}$ & $\begin{array}{l}0.2 \text { ? } \\
\mathrm{ns}\end{array}$ \\
\hline JV & $\begin{array}{l}-0.148 \\
\mathrm{~ns}\end{array}$ & $-0.589 * *$ & $0.269^{n s}$ & $\begin{array}{l}0.144 \\
\text { ns }\end{array}$ & $\begin{array}{l}0.143 \\
\mathrm{~ns}\end{array}$ & $\begin{array}{l}0.139 \\
\text { ns }\end{array}$ & $\begin{array}{l}0.100 \\
\mathrm{~ns}\end{array}$ & 1 & $\begin{array}{l}0.229 \\
\mathrm{~ns}\end{array}$ & $\begin{array}{l}-0.036 \\
\text { ns }\end{array}$ & $\begin{array}{l}0.148 \\
\text { ns }\end{array}$ & $\begin{array}{l}0.073 \\
\text { ns }\end{array}$ & $\begin{array}{l}0.232 \\
\mathrm{~ns}\end{array}$ & $\begin{array}{l}0.1 \% \\
\mathrm{~ns}\end{array}$ \\
\hline FSOP & $\begin{array}{l}0.287 \\
\mathrm{~ns}\end{array}$ & $0.099^{n s}$ & $0.060^{\mathrm{ns}}$ & $\begin{array}{l}-0.116 \\
\text { ns }\end{array}$ & $\begin{array}{l}-0.123 \\
\text { ns }\end{array}$ & $\begin{array}{l}-0.096 \\
n s\end{array}$ & $0.385^{\star \star}$ & $\begin{array}{l}-0.002 \\
\text { ns }\end{array}$ & 1 & $0.186^{\mathrm{ns}}$ & $\begin{array}{l}-0.172 \\
\mathrm{~ns}\end{array}$ & $\begin{array}{l}0.242 \\
\mathrm{~ns}\end{array}$ & $\begin{array}{l}0.284 \\
\mathrm{~ns}\end{array}$ & $0.3 i$ \\
\hline$P G$ & $0.366^{\star}$ & $0.176^{n s}$ & $\begin{array}{l}-0.121 \\
\mathrm{~ns}\end{array}$ & -0.369 * & $-0.365^{\star}$ & -0.299 * & $\begin{array}{l}0.176 \\
\mathrm{~ns}\end{array}$ & $\begin{array}{l}-0.095 \\
\text { ns }\end{array}$ & $\begin{array}{l}0.212 \\
\mathrm{~ns}\end{array}$ & 1 & $\begin{array}{l}-0.206 \\
\text { ns }\end{array}$ & $0.304^{*}$ & $\begin{array}{l}-0.130 \\
\mathrm{~ns}\end{array}$ & 0.3 \\
\hline $\mathrm{CL}$ & $\begin{array}{l}0.072 \\
\mathrm{~ns}\end{array}$ & $0.007^{\mathrm{ns}}$ & $0.137^{\mathrm{ns}}$ & $\begin{array}{l}0.089 \\
\text { ns }\end{array}$ & $\begin{array}{l}0.091 \\
\mathrm{~ns}\end{array}$ & $\begin{array}{l}-0.146 \\
\mathrm{~ns}\end{array}$ & $\begin{array}{l}0.030 \\
\mathrm{~ns}\end{array}$ & $\begin{array}{l}0.084 \\
\mathrm{~ns}\end{array}$ & $\begin{array}{l}-0.088 \\
\text { ns }\end{array}$ & $\begin{array}{l}-0.217 \\
\mathrm{~ns}\end{array}$ & 1 & $\begin{array}{l}-0.015 \\
\mathrm{~ns}\end{array}$ & $0.428 * \star$ & $\begin{array}{l}-0.1 \\
\mathrm{~ns}\end{array}$ \\
\hline CWid & $\begin{array}{l}-0.069 \\
\text { ns }\end{array}$ & $\begin{array}{l}-0.007 \\
\mathrm{~ns}\end{array}$ & $0.01^{\mathrm{ns}}$ & $\begin{array}{l}0.076 \\
\mathrm{~ns}\end{array}$ & $\begin{array}{l}0.074 \\
\mathrm{~ns}\end{array}$ & $\begin{array}{l}-0.136 \\
\mathrm{~ns}\end{array}$ & $0.318^{*}$ & $\begin{array}{l}0.218 \\
\mathrm{~ns}\end{array}$ & $0.452^{\star \star}$ & $0.097^{\mathrm{ns}}$ & $0.321^{\star}$ & 1 & $\begin{array}{l}-0.112 \\
\text { ns }\end{array}$ & $\begin{array}{l}0.0 ! \\
\mathrm{ns}\end{array}$ \\
\hline $\mathrm{CW}$ & $\begin{array}{l}-0.036 \\
\text { ns }\end{array}$ & $0.094^{n s}$ & $\begin{array}{l}-0.175 \\
\mathrm{~ns}\end{array}$ & $0.339 *$ & $0.342^{*}$ & $\begin{array}{l}-0.053 \\
n s\end{array}$ & $0.303^{*}$ & $\begin{array}{l}0.279 \\
\mathrm{~ns}\end{array}$ & $\begin{array}{l}0.249 \\
\mathrm{~ns}\end{array}$ & $\begin{array}{l}-0.179 \\
\mathrm{~ns}\end{array}$ & $0.454^{\star *}$ & $0.585^{\star \star}$ & 1 & $\begin{array}{l}-0.0 \\
\mathrm{~ns}\end{array}$ \\
\hline FSCP & $\begin{array}{l}0.242 \\
\mathrm{~ns}\end{array}$ & $0.137^{n s}$ & $\begin{array}{l}-0.141 \\
\text { ns }\end{array}$ & $\begin{array}{l}0.011 \\
\mathrm{~ns}\end{array}$ & $\begin{array}{l}0.008 \\
\mathrm{~ns}\end{array}$ & $\begin{array}{l}-0.106 \\
\mathrm{~ns}\end{array}$ & $0.489 * *$ & $\begin{array}{l}-0.083 \\
\text { ns }\end{array}$ & $0.582^{\star \star}$ & $0.380 * \star$ & $\begin{array}{l}-0.202 \\
\text { ns }\end{array}$ & $\begin{array}{l}0.221 \\
\mathrm{~ns}\end{array}$ & $\begin{array}{l}0.166 \\
\mathrm{~ns}\end{array}$ & 1 \\
\hline
\end{tabular}

TSS: Total soluble solids, TA: Titratable acidity, BW: Berry weight, FW: Flesh weight, SSW: Single seed weight, SN: Seed number, JV: Juice volume, FSOP: Fruit set in open pollination, PG: Pollen germination, CL: Cluster length, Cwi: Cluster width, CW: Cluster weight, FSCP: Fruit set under controlled pollination.

Marker-trait analyses. A total of 199 alleles were amplified with 39 SSR primer pairs with an average of 5.10 alleles/locus. To identify the level of polymorphism, amount of PIC was calculated for each of the 39 SSR loci which ranged from 0.08 for locus Vmc7c3 to 0.89 for locus VVMD8 with an average value of 0.51 . The observed heterozygosity ranged from 0.083 for locus Vmc7c3 to 0.98 for locus ZAG64 with an average of 0.56 across the SSR markers (Table 3). The studied genotypes were grouped in four main groups based on SSR data (Fig. 1). Hierarchical groupings of cultivars by phenotypic and genotypic data were compared and it was found that $67 \%$ of the cultivars were grouped into similar position across the two hierarchical clusters (Fig. 1 ). The genetic structure of studied grape population were also analyzed with Bayesian clustering method. Results revealed two possible subpopulations ( $\mathrm{K}=2$ ) in the studied germplasm (Fig. 2). Based on the Barplot (Fig. 2), from 45 studied cultivars, 34 cultivars (75.56\%) were belonged to sub-population 1 and 8 cultivars (17.78\%) to sub-population 2. Three cultivars (6.67\%) with $\mathrm{Q}<0.70$ were assigned admixed (Fig. 2). 
Table 3

Genetic parameters of SSR loci assayed in the characterization of table grape cultivars

\begin{tabular}{|c|c|c|c|c|c|c|c|}
\hline Markers & Primer sequence $(5 \rrbracket \rightarrow 3 \triangle)$ & Annealing temperature $\left(C^{\circ}\right)$ & Genotype No & Allele No & Gene Diversity & Heterozygosity & PIC \\
\hline ZAG 62 & $\begin{array}{l}\text { ggtgaaatgggcaccgaacacacgc } \\
\text { ccatgtctctcctcagcttctcagc }\end{array}$ & 53 & 14 & 8 & 0.71 & 0.73 & 0.68 \\
\hline VVS2 & $\begin{array}{l}\text { cagcccgtaaatgtatccatc } \\
\text { aaattcaaaattctaattcaactgg }\end{array}$ & 53 & 22 & 10 & 0.86 & 0.95 & 0.85 \\
\hline VVMD5 & $\begin{array}{l}\text { ctagagctacgccaatccaa } \\
\text { tataccaaaaatcatattcctaaa }\end{array}$ & 56 & 21 & 10 & 0.83 & 0.78 & 0.81 \\
\hline VVMD7 & $\begin{array}{l}\text { agagttgcggagaacaggat } \\
\text { cgaaccttcacacgcttgat }\end{array}$ & 54 & 15 & 6 & 0.80 & 0.78 & 0.77 \\
\hline G7 & $\begin{array}{l}\text { caacagaattcaaatgaaatgga } \\
\text { caaacagcataaatacacaagca }\end{array}$ & 50 & 26 & 14 & 0.90 & 0.93 & 0.89 \\
\hline G10 & $\begin{array}{l}\text { catcattcatccaaattatgtag } \\
\text { tttagtaggttagggataccagt }\end{array}$ & 51 & 7 & 5 & 0.58 & 0.40 & 0.50 \\
\hline D12 & $\begin{array}{l}\text { ctctcttttccgaaattggggt } \\
\text { attttccctggaaacaaagtgg }\end{array}$ & 54 & 18 & 10 & 0.75 & 0.91 & 0.71 \\
\hline $\mathrm{UCH} 29$ & $\begin{array}{l}\text { aaacatgatctgatgcaggtga } \\
\text { caacctgttgatgaaagggaaa }\end{array}$ & 62.5 & 16 & 9 & 0.74 & 0.71 & 0.71 \\
\hline ISV2 & $\begin{array}{l}\text { cactggcctgttgggagataat } \\
\text { ccttcaactggaaaagcctgtc }\end{array}$ & 58 & 17 & 10 & 0.77 & 0.69 & 0.75 \\
\hline ISV3 & $\begin{array}{l}\text { aaggaggagttgagatgtagta } \\
\text { gagtaagagagaagcaagaaaa }\end{array}$ & 58 & 8 & 4 & 0.64 & 0.82 & 0.57 \\
\hline ISV4 & $\begin{array}{l}\text { tgcatagtgctgtaggccattg } \\
\text { tctgtcattgctgtccctttca }\end{array}$ & 59 & 12 & 6 & 0.73 & 0.62 & 0.69 \\
\hline VVS3 & $\begin{array}{l}\text { tgccctatcaattagttcaccta } \\
\text { tcgactttgatatattgatgatt }\end{array}$ & 52 & 3 & 2 & 0.48 & 0.69 & 0.37 \\
\hline VVS4 & $\begin{array}{l}\text { ccatcagtgataaaacctaatgcc } \\
\text { cccaccttgcccttagatgtta }\end{array}$ & 58 & 6 & 4 & 0.46 & 0.44 & 0.42 \\
\hline ZAG 47 & $\begin{array}{l}\text { ggtctcaatacatccgtaagtatat } \\
\text { acggtgtgctctcattgtcattgac }\end{array}$ & 54 & 11 & 6 & 0.75 & 0.84 & 0.72 \\
\hline ZAG64 & $\begin{array}{l}\text { tatgaaagaaacccaacgcggcacg } \\
\text { tgcaatgtggtcagcctttgatggg }\end{array}$ & 58 & 17 & 10 & 0.83 & 0.98 & 0.81 \\
\hline ZAG83 & $\begin{array}{l}\text { ggcggaggcggtagatgagagggcg } \\
\text { acgcaacggctagtaaatacaacgg }\end{array}$ & 52 & 3 & 2 & 0.41 & 0.22 & 0.33 \\
\hline VVMD8 & $\begin{array}{l}\text { taacaaacaagaagaggaat } \\
\text { agcacatccacaacataatg }\end{array}$ & 56 & 20 & 12 & 0.84 & 0.89 & 0.82 \\
\hline VVMD17 & $\begin{array}{l}\text { tgactcgccaaaatctgacg } \\
\text { cacacatatcatcaccacacgg }\end{array}$ & 56 & 6 & 4 & 0.51 & 0.51 & 0.44 \\
\hline VVMD21 & $\begin{array}{l}\text { ggttgtctatggagttgatgttgc } \\
\text { gcttcagtaaaaagggattgcg }\end{array}$ & 56 & 7 & 5 & 0.66 & 0.82 & 0.59 \\
\hline VVMD25 & $\begin{array}{l}\text { ttccgttaaagcaaaagaaaaagg } \\
\text { ttggatttgaaatttattgagggg }\end{array}$ & 56 & 14 & 7 & 0.78 & 0.76 & 0.75 \\
\hline VVMD26 & $\begin{array}{l}\text { gagacgactggtgacattgagc } \\
\text { ccatcaccaccatttctactgc }\end{array}$ & 56 & 5 & 4 & 0.52 & 0.60 & 0.41 \\
\hline
\end{tabular}




\begin{tabular}{|c|c|c|c|c|c|c|c|}
\hline Markers & Primer sequence $(5 \rrbracket \rightarrow 3 \rrbracket)$ & Annealing temperature $\left(\mathrm{C}^{\circ}\right)$ & Genotype No & Allele No & Gene Diversity & Heterozygosity & $\mathrm{PIC}$ \\
\hline VVMD27 & $\begin{array}{l}\text { gtaccagatctgaatacatccgtaagt } \\
\text { acgggtatagagcaaacggtgt }\end{array}$ & 56 & 10 & 5 & 0.72 & 0.78 & 0.67 \\
\hline VVMD32 & $\begin{array}{l}\text { tatgatttttaggggggtgagg } \\
\text { ggaaagatgggatgactcgc }\end{array}$ & 59 & 14 & 8 & 0.71 & 0.71 & 0.67 \\
\hline Scu8vv & $\begin{array}{l}\text { cgagacccagcatcgtttcaag } \\
\text { gcaaaatcctccccgtacaagtc }\end{array}$ & 56 & 2 & 2 & 0.12 & 0.13 & 0.12 \\
\hline Scu10vv & $\begin{array}{l}\text { tacccccacaaccctttt } \\
\text { ttctccgccacctccttttcac }\end{array}$ & 56 & 2 & 2 & 0.37 & 0.49 & 0.30 \\
\hline UDV047 & $\begin{array}{l}\text { tgtatgataatccataatgtgc } \\
\text { taggcatgcttgacttattc }\end{array}$ & 50 & 3 & 2 & 0.50 & 0.33 & 0.38 \\
\hline VMC6E10 & $\begin{array}{l}\text { ctaggtgtgccaagagatcaga } \\
\text { catttgtgggtagttgtgagga }\end{array}$ & 53 & 5 & 3 & 0.61 & 0.38 & 0.53 \\
\hline VMC7a4 & $\begin{array}{l}\text { taaggtggattagtttgggtc } \\
\text { aaactccaaacgatctgattct }\end{array}$ & 51 & 6 & 4 & 0.73 & 0.13 & 0.68 \\
\hline Vmc7b1 & $\begin{array}{l}\text { cacgcaatctctcatttcacaaa } \\
\text { tggtttaggtgacccaaccttta }\end{array}$ & 55 & 3 & 2 & 0.50 & 0.33 & 0.37 \\
\hline Vmc7c3 & $\begin{array}{l}\text { ctttggagagtttccagaggta } \\
\text { actgctctaacagtcctttgct }\end{array}$ & 51 & 2 & 2 & 0.08 & 0.08 & 0.08 \\
\hline Vmc7f2 & $\begin{array}{l}\text { aagaaagtttgcagtttatggtg } \\
\text { aagatgacaatagcgagagagaa }\end{array}$ & 51 & 2 & 2 & 0.29 & 0.36 & 0.25 \\
\hline Vmc7f6 & $\begin{array}{l}\text { attgcttccaaaaagaga } \\
\text { acccaaacccaaatagat }\end{array}$ & 50 & 3 & 2 & 0.19 & 0.17 & 0.17 \\
\hline Vmcng2b7.2 & $\begin{array}{l}\text { tttggagtgaatagagacccct } \\
\text { cagaatttggctccatatttgaa }\end{array}$ & 54 & 4 & 3 & 0.40 & 0.38 & 0.33 \\
\hline Vmcng2h7 & $\begin{array}{l}\text { acgttaaatagaacatggtccc } \\
\text { caacctctttttttgaggtagc }\end{array}$ & 51 & 6 & 3 & 0.63 & 0.68 & 0.56 \\
\hline VVIV67 & $\begin{array}{l}\text { aacttgattgaacaaaggccta } \\
\text { tattatgcctatccagtttcga }\end{array}$ & 50 & 3 & 2 & 0.37 & 0.39 & 0.30 \\
\hline VVIV67.2 & $\begin{array}{l}\text { attctcatttgggttctcac } \\
\text { ttcagtagtcactctcaac }\end{array}$ & 50 & 3 & 2 & 0.45 & 0.60 & 0.35 \\
\hline VVs16 & $\begin{array}{l}\text { tcaaactattattcaaaccaaagtac } \\
\text { tcgatttcaacaaatttagaaata }\end{array}$ & 51 & 3 & 3 & 0.11 & 0.12 & 0.11 \\
\hline VVs29 & $\begin{array}{l}\text { ccccaaggctctgaaaacaat } \\
\text { tgcaaagcaaataaagcttcca }\end{array}$ & 56 & 2 & 2 & 0.45 & 0.68 & 0.35 \\
\hline UDV015 & $\begin{array}{l}\text { tgcacatttccctccttag } \\
\text { cgggttactgggaagggtat }\end{array}$ & 53 & 3 & 2 & 0.27 & 0.15 & 0.23 \\
\hline Mean & & & 8.82 & 5.10 & 0.57 & 0.56 & 0.51 \\
\hline
\end{tabular}

The $r^{2}$ values among SSR markers showed an average value of $20.37\left(r^{2} \geq 0.1\right.$, P-value $\left.\leq 0.01\right)$ (Fig. 2). Using mixed linear model, seven loci found to be significantly associated with the studied traits at $5 \%$ probability level (Table 4). One locus (Scu8vv) was identified to be associated with total soluble solids, one locus (Vmc7f2) with pollen germination, one locus (VVMD17) with seed weight, one locus (Vmc7f2) with cluster weight, one locus (Scu8vv) with fruit set in open pollination, one locus (ISV3) with seed number and one locus (Udv015) with titratable acidity. Results showed that Scu8vv was common for total soluble solids and fruit set in open pollination and also Vmc7f2 was common for pollen germination and cluster weight (Table 4). 
Table 4

SSR loci linked to the studied morphological traits in the analyzed grapevine germplasm using mixed linear model (MLM) procedure

\begin{tabular}{|llllllll|}
\hline Trait & SSR locus & F-marker & P-marker & Trait & SSR locus & F-marker & P-marker \\
\hline Total Soluble Solids & Scu8vv & 5.69 & 0.022 & Cluster weight (g) & Vmc7f2 & 4.86 & 0.033 \\
\hline Pollen germination (\%) & Vmc7f2 & 5.08 & 0.029 & Fruit set in open pollination (\%) & Scu8vv & 4.66 & 0.037 \\
\hline Seed weight (g) & VVMD17 & 2.76 & 0.032 & Seed number & ISV3 & 2.44 & 0.038 \\
& & & & Titratable acidity & Udv015 & 3.47 & 0.044 \\
\hline
\end{tabular}

Predicting breeding values. The highest (2.4342) and the lowest (-2.976) breeding value for total soluble solids (TSS) was observed in "KIkarevi" and "At Ouzum" cultivars, respectively. The highest (0.2052) and lowest (-0.0773) breeding value for pH was observed in "Tabarze Qermez" and "Garmian", respectively. The highest (0.0942) and lowest (-0.0645) breeding value for TA character was seen in "KIkarevi" and "Shirazi" cultivars, respectively. For berry weight, the highest breeding value (1.4583) was observed in "Qzl Ouzum" and the lowest one (-2.9236) was observed in "Rejin". The highest breeding value (1.4914) for flesh weight was observed in "Qzl Ouzum" and the lowest one (-2.4007) was seen in "Rejin". About single seed weight, the highest breeding value (0.0521) was seen in "Shirazi" and the lowest one (-0.309) was observed in "Askari". The highest $(0.6241)$ and lowest ( 0.5183$)$ breeding value for seed number was observed in "Chava Ga" and "Mam Braima" cultivars. For juice volume, the highest breeding value (4.6295) was seen in "Sahebi Qermez" and the lowest one (-4.5384) was observed in "Agh Shani" cultivar. The highest (17.8417) and lowest (-19.7354) breeding value for fruit set in open pollination was observed in "Qara Shani" and "Mam Braima" cultivars. Concerning to pollen germination, the highest (24.9031) and lowest (-20.3949) breeding value were observed in "Fakhri" and "Mam Braima" cultivars, respectively. The highest breeding value (2.8615) for cluster length was observed in "Qzl Ouzum" and the lowest one (-3.3038) was seen in "Lal Sefid" cultivar. The highest breeding value (2.2685) for cluster width was observed in "Lal Qermez" and the lowest one $(-1.3292)$ was seen in "Mam Braima" cultivar. About cluster weight, the highest (211.783) and lowest (-121.986) breeding value were observed in "Shahroudi" and "Yaghoti" cultivars, respectively. For fruit set under controlled pollination, the highest (9.1738) and lowest (-9.7768) breeding value were observed in "Qara Shani" and "Bol Mazu" cultivars, respectively (Table 5).

Considering the breeding values of all studied traits, "Taifi”, “Qzl Ouzum”, “Rishbaba Qermez”, “Garmian”, “Agh Shani”, "Lal Qermez”, “Sahebi Qermez”, "Saghal Solian" cultivars were top ranked and "Askari", "Hosseini", "Inah Amjai", "Goi Melki”, "Mam Braima”, "Sachakh" and "Agh Melhi” cultivars got the worst ranking (Table 5). Narrow sense heritability was calculated for all studied traits based on predicted breeding values. Narrow sense heritabilities were $46.21 \%, 3.83 \%$ and $1.97 \%$ for total soluble solids (TSS), $\mathrm{pH}$ and titratable acidity (TA), respectively. For berry and flesh weights, it was estimated around $88.51 \%$ and $83.51 \%$, respectively. For seed weight and seed number narrow sense heritabilities were $32.13 \%$ and $32.33 \%$. For juice volume it was estimated $17.46 \%$. Concerning to fruit set in open pollination and fruit set under controlled pollination narrow sense heritabilities were estimated $38.25 \%$ and $15.37 \%$, respectively. It was $46.19 \%$ for pollen germination. For cluster length, cluster width and cluster weight narrow sense heritabilities were estimated $25.37 \%$, $9.32 \%$ and $27.34 \%$, respectively (Table 5). The highest heritability was observed in berry and flesh weights and the lowest one was seen in TA, pH and cluster width characters (Table 5). 
Table 5

Breeding value of $V$. vinifera cultivars in studying traits

\begin{tabular}{|c|c|c|c|c|c|c|c|c|c|c|c|c|c|c|}
\hline Number & Cultivar & TSS & Rank & $\mathrm{pH}$ & Rank & TA & Rank & BW & Rank & FW & Rank & ssw & Rank & SN \\
\hline 1 & Rezghi & -0.6385 & 21 & -0.0075 & 14 & 0.0168 & 39 & -1.0902 & 12 & -1.0133 & 12 & -0.0041 & 25 & -0.2511 \\
\hline 2 & Hosseini & -2.4015 & 5 & -0.0085 & 13 & -0.0373 & 15 & 0.2769 & 30 & 0.4187 & 31 & -0.0116 & 10 & -0.2902 \\
\hline 3 & $\begin{array}{l}\text { Tabarze } \\
\text { Sefid }\end{array}$ & 1.5597 & 40 & 0.1891 & 44 & -0.0549 & 5 & -0.8497 & 15 & -0.8001 & 15 & -0.0095 & 16 & -0.1594 \\
\hline 4 & $\begin{array}{l}\text { Saghal } \\
\text { Solian }\end{array}$ & 1.0926 & 34 & 0.1799 & 42 & -0.0463 & 8 & 0.0462 & 28 & 0.1245 & 28 & -0.0031 & 26 & 0.0325 \\
\hline 5 & At Ouzum & $-2.9760 *$ & 1 & -0.0643 & 3 & 0.0023 & 33 & 0.6809 & 39 & 0.7630 & 38 & -0.0098 & 15 & 0.4067 \\
\hline 6 & Lal Seyah & 0.5610 & 32 & -0.0219 & 10 & -0.0113 & 28 & -0.6362 & 19 & -0.6202 & 18 & 0.0028 & 34 & 0.3193 \\
\hline 7 & $\begin{array}{l}\text { Seyah } \\
\text { Sardasht }\end{array}$ & 1.2420 & 36 & 0.0183 & 19 & -0.0045 & 30 & $-1.7427 * \star$ & 2 & -1.6217 ** & 2 & -0.0110 & 13 & 0.4132 \\
\hline 8 & Garmian & -0.2857 & 23 & -0.0773 & 1 & 0.0219 & 41 & 0.7280 & 40 & 0.7869 & 41 & -0.0049 & 23 & 0.2872 \\
\hline 9 & Maiemo & -0.9128 & 16 & -0.0143 & 11 & 0.0044 & 35 & -1.1177 & 11 & -0.9748 & 13 & -0.0094 & 17 & -0.1049 \\
\hline 10 & $\begin{array}{l}\text { Rishbaba } \\
\text { Qermez }\end{array}$ & 0.2513 & 30 & 0.0415 & 26 & -0.0293 & 19 & -0.8159 & 16 & -0.6876 & 17 & -0.0075 & 21 & 0.3178 \\
\hline 11 & Taifi & -0.0364 & 25 & -0.0122 & 12 & -0.0402 & 12 & 0.6112 & 36 & 0.6477 & 36 & 0.0029 & 35 & $0.6065^{\circ}$ \\
\hline 12 & $\begin{array}{l}\text { Keshmeshi } \\
\text { Qermez }\end{array}$ & 1.3684 & 38 & 0.1285 & 39 & -0.0159 & 26 & $-1.1752^{*}$ & 10 & $-1.1400 *$ & 10 & $-0.0224^{\star}$ & 3 & -0.1837 \\
\hline 13 & Fakhri & 2.0662 & 44 & 0.0929 & 35 & -0.0639 & 2 & -0.5695 & 20 & -0.4567 & 21 & 0.0014 & 32 & -0.3179 \\
\hline 14 & Shahroudi & -2.4115 & 4 & 0.0781 & 32 & -0.0308 & 18 & -0.4257 & 24 & -0.3069 & 25 & -0.0101 & 14 & -0.2643 \\
\hline 15 & $\begin{array}{l}\text { Qara } \\
\text { Shani }\end{array}$ & 1.2783 & 37 & -0.0431 & 5 & 0.0592 & 44 & -0.3569 & 25 & -0.3220 & 24 & -0.0087 & 19 & $0.5828^{\prime}$ \\
\hline 16 & $\begin{array}{l}\text { Sahebi } \\
\text { Qermez }\end{array}$ & -1.0808 & 13 & 0.0186 & 20 & -0.0225 & 23 & 0.9778 & 42 & 1.0421 & 42 & -0.0024 & 27 & 0.1786 \\
\hline 17 & Inah Amjai & -2.1367 & 8 & 0.0395 & 25 & -0.0559 & 4 & 0.3703 & 33 & 0.4735 & 34 & -0.0067 & 22 & -0.2860 \\
\hline 18 & $\begin{array}{l}\text { Tabarze } \\
\text { Qermez }\end{array}$ & 1.3766 & 39 & 0.2052 & 45 & -0.0382 & 14 & -0.5135 & 21 & -0.5489 & 20 & -0.0128 & 9 & -0.2468 \\
\hline 19 & Dastarchin & 1.0645 & 33 & 0.1732 & 41 & -0.0209 & 24 & -0.7601 & 17 & -0.7148 & 16 & -0.0116 & 10 & -0.3113 \\
\hline 20 & $\begin{array}{l}\text { Rishbaba } \\
\text { Sefid }\end{array}$ & -1.5506 & 11 & 0.0068 & 17 & 0.0027 & 34 & $-1.7275^{\star \star}$ & 4 & $-1.5865^{\star \star}$ & 3 & -0.0081 & 20 & -0.2170 \\
\hline 21 & Agh Melhi & 0.0413 & 28 & -0.0352 & 6 & -0.0003 & 32 & $-1.3364^{*}$ & 8 & -1.1931 & 9 & -0.0139 & 8 & -0.1902 \\
\hline 22 & Goi Melki & -1.6139 & 10 & 0.0370 & 24 & -0.0429 & 10 & 0.3337 & 32 & 0.4207 & 32 & 0.0091 & 41 & -0.2937 \\
\hline 23 & Sayani & -0.7039 & 19 & 0.0208 & 21 & -0.0466 & 7 & -1.0822 & 13 & -0.9724 & 14 & 0.0008 & 31 & -0.1426 \\
\hline 24 & Kalati & -1.5012 & 12 & 0.0476 & 28 & -0.0261 & 21 & -0.1787 & 26 & -0.0832 & 27 & -0.0048 & 24 & -0.0261 \\
\hline 25 & $\begin{array}{l}\text { Mam } \\
\text { Braima }\end{array}$ & -2.4737 & 3 & 0.0736 & 31 & -0.0156 & 27 & 0.7358 & 41 & 0.7742 & 40 & $0.0279 *$ & 44 & -0.5183 \\
\hline 26 & Bol Mazu & -0.4542 & 22 & -0.0282 & 9 & 0.0113 & 37 & 0.6793 & 38 & 0.7728 & 39 & 0.0024 & 33 & -0.2109 \\
\hline 27 & $\begin{array}{l}\text { Lal } \\
\text { Qermez }\end{array}$ & -1.0505 & 14 & 0.0512 & 30 & -0.0489 & 6 & 0.1149 & 29 & 0.1475 & 29 & -0.0015 & 28 & 0.5315 \\
\hline 28 & $\begin{array}{l}\text { Sefid } \\
\text { Shakh } \\
\text { Shakh }\end{array}$ & 1.6054 & 41 & 0.1485 & 40 & -0.0240 & 22 & $-1.4358^{\star}$ & 6 & $-1.2961^{\star}$ & 6 & $-0.0222^{\star}$ & 4 & -0.1006 \\
\hline 29 & Alhaghi & -2.4973 & 2 & 0.1064 & 38 & -0.0420 & 11 & -0.7402 & 18 & -0.6033 & 19 & -0.0140 & 7 & -0.4194 \\
\hline 30 & Askari & -2.1175 & 9 & 0.0436 & 27 & 0.0217 & 40 & $-1.7321^{\star \star}$ & 3 & $-1.5386^{*}$ & 4 & $-0.0309 *$ & 1 & -0.2336 \\
\hline 31 & $\begin{array}{l}\text { Keshmeshi } \\
\text { Sefid }\end{array}$ & 1.8917 & 43 & 0.0995 & 36 & -0.0181 & 25 & $-1.4123^{\star}$ & 7 & $-1.2717^{*}$ & 7 & -0.0229 & 2 & -0.1229 \\
\hline 32 & Rejin & 1.8828 & 42 & 0.1890 & 43 & -0.0278 & 20 & $-2.9236^{\star \star}$ & 1 & $-2.4007 * \star$ & 1 & -0.0187 & 5 & -0.0825 \\
\hline 33 & Sarghola & 0.0062 & 27 & -0.0310 & 7 & -0.0600 & 3 & $1.2493^{*}$ & 43 & $1.3487^{\star}$ & 44 & -0.0008 & 30 & 0.4917 \\
\hline 34 & Chava Ga & 0.3101 & 31 & -0.0731 & 2 & 0.0082 & 36 & 0.5232 & 35 & 0.4403 & 33 & 0.0128 & 42 & $0.6241^{\prime}$ \\
\hline 35 & Yaghoti & -0.1852 & 24 & -0.0029 & 16 & 0.0499 & 43 & $-1.3076^{\star}$ & 9 & $-1.2099 *$ & 8 & 0.0089 & 40 & -0.3102 \\
\hline
\end{tabular}




\begin{tabular}{|c|c|c|c|c|c|c|c|c|c|c|c|c|c|c|}
\hline Number & Cultivar & TSS & Rank & $\mathrm{pH}$ & Rank & TA & Rank & BW & Rank & FW & Rank & ssW & Rank & SN \\
\hline 36 & $\begin{array}{l}\text { Qara } \\
\text { Gandoma }\end{array}$ & -0.9326 & 15 & 0.0221 & 22 & -0.0028 & 31 & -0.4893 & 23 & -0.4284 & 22 & 0.00881 & 39 & -0.1675 \\
\hline 37 & Gazandaii & -2.2117 & 7 & -0.0045 & 15 & -0.0326 & 17 & $1.2770 *$ & 44 & $1.3305^{\star}$ & 43 & -0.0093 & 18 & 0.2204 \\
\hline 38 & Qzl Ouzum & 0.1568 & 29 & 0.1033 & 37 & -0.0456 & 9 & $1.4583^{\star}$ & 45 & $1.4914^{\star}$ & 45 & 0.0080 & 37 & 0.1409 \\
\hline 39 & Agh Shani & 1.1959 & 35 & 0.0171 & 18 & 0.0148 & 38 & 0.3055 & 31 & 0.4025 & 30 & -0.0112 & 12 & 0.0642 \\
\hline 40 & Jig Jiga & -0.7944 & 17 & -0.0461 & 4 & 0.0308 & 42 & -1.0584 & 14 & -1.0549 & 11 & 0.0080 & 37 & 0.2285 \\
\hline 41 & Lal Sefid & -0.6458 & 20 & 0.0282 & 23 & -0.0051 & 29 & -0.5030 & 22 & -0.3747 & 23 & -0.0162 & 6 & -0.0306 \\
\hline 42 & Klkarevi & 2.4342 & 45 & -0.0287 & 8 & 0.0942 & 45 & 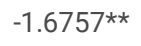 & 5 & $-1.5302^{*}$ & 5 & -0.0012 & 29 & -0.4108 \\
\hline 43 & Sachakh & -0.7598 & 18 & 0.0499 & 29 & -0.0341 & 16 & 0.4987 & 34 & 0.5552 & 35 & 0.0139 & 43 & -0.3665 \\
\hline 44 & Shirazi & -2.3412 & 6 & 0.0906 & 34 & -0.0645 & 1 & -0.0194 & 27 & -0.0927 & 26 & $0.0521^{\star *}$ & 45 & -0.2433 \\
\hline \multirow[t]{2}{*}{45} & Angotka & -0.0311 & 26 & 0.0818 & 33 & -0.0400 & 13 & 0.6746 & 37 & 0.7053 & 37 & 0.0071 & 36 & 0.2513 \\
\hline & $\begin{array}{l}\% \text { Narrow } \\
\text { sense } \\
\text { heritability }\end{array}$ & 46.21 & & 3.83 & & 1.97 & & 88.51 & & 83.51 & & 32.13 & & 32.33 \\
\hline
\end{tabular}


Table 5

(Continuation) Breeding value of $V$. vinifera cultivars in studying traits

\begin{tabular}{|c|c|c|c|c|c|c|c|c|c|c|c|c|c|c|}
\hline Number & Cultivar & JV & Rank & FSOP & Rank & PG & Rank & $\mathrm{CL}$ & Rank & CWi & Rank & $\mathrm{CW}$ & Rank & $\mathrm{FSCl}$ \\
\hline 1 & Rezghi & 2.8965 & 42 & $-12.5984^{*}$ & 3 & -8.1994 & 8 & 2.3681 & 41 & 0.0536 & 22 & -31.423 & 16 & $-4.7 \epsilon$ \\
\hline 2 & Hosseini & -0.8817 & 20 & -9.5757 & 6 & 3.9173 & 22 & -3.1628 & 3 & -0.9271 & 3 & -78.435 & 4 & $1.30^{\prime}$ \\
\hline 3 & $\begin{array}{l}\text { Tabarze } \\
\text { Sefid }\end{array}$ & $-3.8019 *$ & 3 & -8.5253 & 9 & 12.5161 & 37 & 0.0463 & 15 & -0.2859 & 13 & -37.536 & 15 & 1.59 \\
\hline 4 & $\begin{array}{l}\text { Saghal } \\
\text { Solian }\end{array}$ & -0.6768 & 22 & -6.4190 & 18 & 16.0167 & 40 & 0.8406 & 27 & 0.4573 & 35 & 23.029 & 34 & 2.32 \\
\hline 5 & At Ouzum & 0.7094 & 36 & -5.4238 & 22 & -0.1167 & 17 & 0.9101 & 30 & 0.0831 & 26 & 16.473 & 32 & -0.93 \\
\hline 6 & Lal Seyah & -2.0783 & 12 & 8.0225 & 43 & -4.9109 & 14 & -2.1832 & 6 & -0.8408 & 5 & 4.068 & 28 & 7.82 \\
\hline 7 & $\begin{array}{l}\text { Seyah } \\
\text { Sardasht }\end{array}$ & -0.1224 & 29 & -6.2370 & 19 & 8.1469 & 28 & -1.6229 & 7 & -0.4276 & 11 & -65.036 & 6 & 1.30: \\
\hline 8 & Garmian & -1.2243 & 17 & 0.3276 & 34 & 8.8130 & 31 & 0.7535 & 25 & 0.6913 & 39 & 37.042 & 37 & 8.72 \\
\hline 9 & Maiemo & 1.7818 & 38 & -9.3284 & 7 & 2.4785 & 19 & 1.6377 & 35 & 0.7302 & 40 & -31.235 & 17 & -4.27 \\
\hline 10 & $\begin{array}{l}\text { Rishbaba } \\
\text { Qermez }\end{array}$ & 0.3599 & 33 & 2.4234 & 37 & $22.5191^{*}$ & 43 & 2.2945 & 38 & 1.3263 & 43 & 35.210 & 36 & 3.24: \\
\hline 11 & Taifi & 3.3511 & 44 & -1.7388 & 30 & 4.9552 & 24 & 0.0794 & 16 & 0.7582 & 41 & $171.652^{*}$ & 42 & 3.47 \\
\hline 12 & $\begin{array}{l}\text { Keshmeshi } \\
\text { Qermez }\end{array}$ & $-3.4054^{\star}$ & 5 & -8.2283 & 10 & 8.2316 & 30 & 0.3833 & 21 & -0.0931 & 18 & -40.898 & 14 & $1.51:$ \\
\hline 13 & Fakhri & -2.2115 & 11 & 2.8535 & 40 & $24.9031^{\star * *}$ & 45 & -2.3826 & 4 & 0.0556 & 23 & -108.296 & 2 & 1.25 \\
\hline 14 & Shahroudi & -0.2711 & 25 & 1.7806 & 36 & -8.0582 & 9 & 2.5863 & 43 & 0.0758 & 25 & $211.783^{*}$ & 45 & $-3.9 C$ \\
\hline 15 & $\begin{array}{l}\text { Qara } \\
\text { Shani }\end{array}$ & -0.1970 & 28 & $17.8417^{\star \star *}$ & 45 & -5.4311 & 12 & -0.4402 & 10 & 0.1703 & 28 & -47.266 & 12 & 9.17 \\
\hline 16 & $\begin{array}{l}\text { Sahebi } \\
\text { Qermez }\end{array}$ & $4.6295^{\star}$ & 45 & -0.3362 & 32 & -13.8949 & 4 & 0.8705 & 28 & $2.0291^{*}$ & 44 & $174.977^{\star}$ & 43 & -1.23 \\
\hline 17 & Inah Amjai & -1.0278 & 18 & -8.1471 & 11 & 4.9079 & 23 & -3.2069 & 2 & -0.6583 & 8 & -49.957 & 10 & 0.61 \\
\hline 18 & $\begin{array}{l}\text { Tabarze } \\
\text { Qermez }\end{array}$ & -3.3934 & 6 & -6.0394 & 20 & 11.5504 & 33 & 1.1631 & 34 & 0.3336 & 32 & -2.446 & 26 & 1.32 \\
\hline 19 & Dastarchin & -2.5875 & 9 & -7.6361 & 14 & 10.1940 & 32 & 1.1442 & 31 & 0.3937 & 34 & -9.197 & 24 & 0.50 \\
\hline 20 & $\begin{array}{l}\text { Rishbaba } \\
\text { Sefid }\end{array}$ & 2.7125 & 40 & -7.2191 & 16 & 8.2271 & 29 & 2.3439 & 40 & 0.6698 & 38 & -26.010 & 20 & -4.77 \\
\hline 21 & Agh Melhi & 0.4595 & 34 & -4.0021 & 27 & 16.0685 & 41 & -1.5739 & 8 & -0.5244 & 9 & -85.569 & 3 & $-0.8 \mathrm{C}$ \\
\hline 22 & Goi Melki & -0.3369 & 24 & $-12.5808^{*}$ & 4 & 6.0848 & 26 & -0.3074 & 11 & -0.8063 & 6 & -75.823 & 5 & $-5.5 £$ \\
\hline 23 & Sayani & 1.7232 & 37 & -5.3235 & 23 & -4.9499 & 13 & 1.1554 & 33 & -0.2325 & 14 & 44.736 & 38 & -3.93 \\
\hline 24 & Kalati & 0.0249 & 30 & 2.4369 & 38 & -9.3695 & 7 & 0.7634 & 26 & 0.6215 & 37 & -29.348 & 19 & $2.11^{\prime}$ \\
\hline 25 & $\begin{array}{l}\text { Mam } \\
\text { Braima }\end{array}$ & -0.3735 & 23 & $-19.7354^{\star \star}$ & 1 & $-20.3949 *$ & 1 & -0.1071 & 13 & -1.3292 & 1 & -57.176 & 9 & $-9.6 \varepsilon$ \\
\hline 26 & Bol Mazu & 2.0746 & 39 & -10.3485 & 5 & -13.6343 & 5 & 0.3451 & 20 & -1.0033 & 2 & -25.115 & 21 & -9.77 \\
\hline 27 & $\begin{array}{l}\text { Lal } \\
\text { Qermez }\end{array}$ & 2.7605 & 41 & -1.3236 & 31 & 11.7650 & 34 & 0.8706 & 29 & $2.2685^{\star}$ & 45 & 101.809 & 41 & $-1.3 \mathrm{C}$ \\
\hline 28 & $\begin{array}{l}\text { Sefid } \\
\text { Shakh } \\
\text { Shakh }\end{array}$ & -3.1198 & 7 & -7.4481 & 15 & $15.2255^{\star}$ & 39 & 0.5322 & 24 & 0.0574 & 24 & -31.013 & 18 & 2.58 \\
\hline 29 & Alhaghi & -0.2127 & 27 & 2.6229 & 39 & -12.5528 & 6 & 2.7001 & 44 & 0.2441 & 30 & $208.685^{\star}$ & 44 & $-2.5 \varepsilon$ \\
\hline 30 & Askari & -3.9114 & 2 & -9.1625 & 8 & 14.0619 & 38 & 0.0066 & 14 & -0.3783 & 12 & -59.188 & 8 & -3.61 \\
\hline 31 & $\begin{array}{l}\text { Keshmeshi } \\
\text { Sefid }\end{array}$ & -1.5355 & 16 & -7.6692 & 12 & $18.4415^{\star}$ & 42 & 0.0946 & 17 & -0.4602 & 10 & -44.847 & 13 & 1.53: \\
\hline 32 & Rejin & -2.5737 & 10 & -4.4204 & 26 & 23.9810 ** & 44 & 0.4288 & 22 & -0.0803 & 19 & -23.599 & 22 & 3.27: \\
\hline 33 & Sarghola & -0.8607 & 21 & -5.1890 & 24 & 3.5831 & 20 & 0.1044 & 18 & 0.3848 & 33 & 23.464 & 35 & -0.34 \\
\hline 34 & Chava Ga & -1.7135 & 14 & -5.6535 & 21 & $-18.3529 *$ & 2 & 0.4452 & 23 & 0.2779 & 31 & 13.879 & 31 & -3.15 \\
\hline
\end{tabular}




\begin{tabular}{|llllllllllllllll}
\hline Number & Cultivar & JV & Rank & FSOP & Rank & PG & Rank & CL & Rank & CWi & Rank & CW & Rank & FSCl \\
\hline 35 & Yaghoti & -2.9675 & 8 & 0.0457 & 33 & 7.2376 & 27 & -0.2837 & 12 & 0.0392 & 21 & -121.986 & 1 & -4.83 \\
\hline 36 & $\begin{array}{l}\text { Qara } \\
\text { Gandoma }\end{array}$ & 3.0149 & 43 & -6.9581 & 17 & -16.8250 & 3 & 1.7657 & 36 & 0.1327 & 27 & -4.943 & 25 & -0.37 \\
\hline 37 & Gazandaii & 0.2445 & 32 & -7.6455 & 13 & -4.6550 & 15 & 2.3883 & 42 & -0.7836 & 7 & 6.706 & 30 & $-0.7 \varepsilon$ \\
\hline 38 & Qzl Ouzum & 0.1929 & 31 & -5.0105 & 25 & -6.9770 & 10 & 2.8615 & 45 & 0.1785 & 29 & 82.887 & 40 & 0.46 \\
\hline 39 & Agh Shani & $-4.5384^{\star}$ & 1 & 3.1126 & 41 & 5.0923 & 25 & 2.2979 & 39 & 1.0296 & 42 & 68.073 & 39 & 5.60 \\
\hline 40 & Jig Jiga & -1.8475 & 13 & -2.5927 & 29 & 1.3384 & 18 & 1.1496 & 32 & 0.4889 & 36 & -47.595 & 11 & 0.28 \\
\hline 41 & Lal Sefid & -3.4780 & 4 & 5.7738 & 42 & 11.8121 & 35 & -3.3038 & 1 & 0.0114 & 20 & 4.649 & 29 & 1.45 \\
\hline 42 & Klkarevi & -0.2225 & 26 & 10.4248 & 44 & -5.5205 & 11 & 2.0237 & 37 & -0.2150 & 15 & 0.944 & 27 & -4.69 \\
\hline 43 & Sachakh & -1.6872 & 15 & $-14.0367 *$ & 2 & -4.2272 & 16 & 0.1425 & 19 & -0.9138 & 4 & -64.211 & 7 & $-5.4 \varepsilon$ \\
\hline 44 & Shirazi & 0.4941 & 35 & 1.2941 & 35 & 12.2134 & 36 & -2.2940 & 5 & -0.1390 & 17 & -20.502 & 23 & 5.78 \\
\hline 45 & Angotka & -0.9521 & 19 & -2.9218 & 28 & 3.7512 & 21 & -0.6779 & 9 & -0.2081 & 16 & 16.540 & 33 & -3.57 \\
\hline & \%Narrow & 17.46 & & 38.25 & & 46.19 & & 25.37 & & 9.32 & & 27.34 & 15.3 \\
& sense \\
heritability & & & & & & & & & & & & \\
\hline
\end{tabular}

TSS: Total soluble solids, TA: Titratable acidity, BW: Berry weight, FW: Flesh weight, SSW: Single seed weight, SN: Seed number, JV: Juice volume, FSOP: Fruit set in open pollination, PG: Pollen germination, CL: Cluster length, Cwi: Cluster width, CW: Cluster weight, FSCP: Fruit set under controlled pollination.

Synteny analysis of distribution of genotypes across dendrograms. Comparing the phenotypic clustering with that produced by estimated breeding values revealed that $26.67 \%$ of cultivars were grouped into the same groups across the two hierarchical clusters (Fig. 1). Cluster analysis by breeding values as well as that based on SSR genotypic data revealed the presence of four genetic groups in the studied germplasm (Fig. 1). It was observed that the genotypes distribution pattern in clustering with breeding values is different with that clustered by SSR genotypic data (Fig. 1). By comparing the two dendrograms, it was found that all of cultivars except "Rishbaba Qermez" in group 1 of molecular cluster were grouped into similar position across hierarchical clustering with breeding values (Fig. 1).

Discriminant analysis of principal components (DAPC). To further clarify the genetic relationship between groups resulting from each one of molecular, morphological and breeding value cluster analyses; discriminant analysis of principal components (DAPC) was performed. DAPC results classified obviously the collections into four groups (Fig. 3). Results of molecular DAPC showed high dissimilarity between groups. However, some similarity was observed between groups 1 and 2 and also between groups 1 and 3 . Density plot of individuals along the first discriminant function also indicated the presence of four groups based on Bayesian information criterion (BIC). High genetic diversity was observed in group 3 . Red and orange colored peaks corresponding to groups 1 and 3 are largely overlap. Blue colored peak corresponding to group 4 is completely distinct. DAPC analysis of morphological and breeding values showed high similarity between groups. Groups 1, 2, 3 and 4 are largely overlap (Fig. 3).

\section{Discussion}

Two subpopulations ( $\mathrm{K}=2)$ were identified in the studied grape germplasm. Based on membership probability of greater than or equal to 70 percent, $75.56 \%$ of cultivars were belonged to population 1 and $17.78 \%$ of cultivars were belonged to population 2 . When the membership probability of a genotype to a cluster is greater than or equal to 0.70 , the genotype is assigned to one of subpopulations but if the membership probability is less than that, it is defined as admixed genotype ${ }^{53}$. The presence of structure in the studied population is a deterrent factor to achieving reliable results. As a result, when population structure and kinship relations exist in association panel and not to be considered in the association analysis, they produce false positive results ${ }^{8}$. Based on $r^{2}$ statistics, some SSR locus pairs showed significant level of linkage disequilibrium. The extend of LD depended on different factors, for example some factors such as small population size, inbreeding, population admixture, genetic draft, autogamy and epistasis increase LD level. On the other hand, allogamy, high recombination rate and high mutation rate decrease LD level ${ }^{1}$. The description of LD was reported in French wild grapes for first time ${ }^{2}$. It was reported that $L D$ level is twelve times higher in native grapes than in wild grapes ${ }^{2}$.

In this study seven SSR loci were found to be significantly associated with genomic regions controlling studied traits. Several QTLs were reported for berry weight, seed number and seed fresh weight in grape and among of these, the one reported for seedlessness was a major effect QTL and closely linked to VMC7F2 locus $^{9}$. In other study close association of two SSR markers (VVIB23 and VVMD34) with flower sex was reported ${ }^{3,39,47}$. Several QTLs for sugar content on LGs 1, 2, 3, 4, 7, 9, 11 and some other for total acid on LGs 06,13 , and 18 were reported ${ }^{10}$. Sugar and total acid are important factors in the taste of grapes. Hexose (Fructose and glucose) are predominant sugars in grape at mature stage ${ }^{52,38}$. Some QTLs were common among different traits. Identification of common markers for the studied traits may be due to linkage or pleiotropic effects ${ }^{36}$. Identification of common markers is important in plant breeding program because it enables simultaneous selection for several traits ${ }^{32,59}$.

Breeding values were estimated for 14 pomological traits in grape cultivars using the best linear unbiased prediction (BLUP). Developing and introducing new cultivars requires parental selection within large germplasm populations, so estimating the value of genotype is an important step in breeding programs.

Page 12/19 
Breeding value is an important notion in plant and animal breeding ${ }^{17}$. He defined breeding value as the mean value of its offspring. Application of BLUP for estimating individual breeding value has been reported in forest tree ${ }^{65,63}$. It was reported using of BLUP for estimating breeding values in peach genotypes ${ }^{13}$. BLUP were applied to predict the general combining ability (GCA) and specific combining ability (SCA) for date of ripening in apples ${ }^{54}$.

The organoleptic quality of table grapes depends on the sugar content, organic acid content and the balance between them. Organic acids are present in a small amount compared to sugars, but it generally plays an important role in the taste ${ }^{42}$. A few cultivars with high-sucrose concentration were reported in Vitis rotundifolia and hybrids between V. labrusca and $V$. vinifera ${ }^{38}$. In this study "Klkarevi" cultivar has the highest breeding value for TSS and TA. Seedlessness is another important and desirable factor for consumers. Development of seeds in grapes are controlled by a1, a2, a3 and i genes. The i gene is a regulator gene. Seedless expression occurs when a1, a2, and a3 are homozygous as recessive, and the regulator gene is homozygous as 'Il' or heterozygous 'li' ${ }^{6}$. In stenospermocarpy grape, fertilization occurs but embryo is aborted in earlier stages and seed trace remains ". Among seedless studied cultivars, "Rejin" and "Askari" cultivars had the highest and lowest breeding value in terms of single seed weight and seed number. Among seeded cultivars, "Shirazi" and "Lal Sefid" cultivars had the highest and lowest breeding value for single seed weight, respectively. For seed number, "Chava Ga" and "Mam Braima" cultivars had the highest and lowest breeding values, respectively.

Pollination is an important factor that affects the percentage of fruit set. It was reported wide percentage of pollen germination from $11.4 \%$ in 'Thompson Seedless to 39.1\% in 'King's Ruby' grapevine cultivars ${ }^{55}$. Among the cultivars investigated in the present study, "Rishbaba Qermez”, "Fakhri”, "Sefid Shakh Shakh", "Keshmeshi Sefid" and "Rejin" cultivars have a high and positive breeding value for pollen germination. Researchers investigated pollen germination in 14 grape cultivars and reported that Touriga Nacional, Cabernet Franc and Cabernet Sauvignon cultivars had low pollen germination while Castelao, Loureiro, Malbec and Petit Verdot cultivars showed high percent of pollen germination ${ }^{44}$.

The end purpose of most breeders is to combine the appropriate situation of more than one trait in common background. From view of estimated breeding values, “Saghal Solian”, “At Ouzum”, Garmian”, “Rishbaba Qermez”, “Taifi”, “Shahroudi”, “Sahebi Qermez”, “Lal Qermez”, "Alhaghi”, “Sarghola”, “Chava Ga”, “Qzl Ouzum" and "Agh Shani" cultivars are suitable parents for cluster length, cluster width and cluster weight improvement. They can be used in hybridization programs because they can better transfer their characteristics to the progeny. The best parent for high berry weight, flesh weight, cluster length, cluster width and cluster weight would be "Sarghola” and "Qzl Ouzum” cultivars. "Garmian”, "Rishbaba Qermez", "Fakhri”, "Agh Shani”, "Lal Sefid” and "Shirazi” cultivars are best parent for breeding pollen germination, fruit set in open pollination and fruit set under controlled pollination characters. "Yaghoti", "Fakhri", "Shirazi", "Rishbaba Sefid", "Rezghi" and "Maiemo" cultivars clustered into the same group in both breeding value and molecular clustering dendrograms. These cultivars have negative breeding values for berry weight, flesh weight and cluster weight characters. The wide range of breeding value for most of evaluated traits and the possibility to choose a genotype in term of good situation of more traits propose that breeders should evaluate the germplasm according to their purpose before selecting best individuals. BLUP is an effective option for achieving to this goal ${ }^{18}$. There is not any cultivar that high-grade breeding value for all traits. The solution is to intercross individuals with positive traits and then select progenies during several generations.

In the selection process based on phenotypic value, success in changing population characteristics is predictable if the degree of conformity between phenotypic and genotypic values to be high. Measuring the degree of conformity is made by calculating heritability ${ }^{18}$. Heritability is influenced by type of trait, studied population, environmental conditions and the method of phenotype measurement ${ }^{20}$. Berry weight and flesh weight showed high heritability and compared to other studied traits are more under control of the additive effects of genes. Researches has shown that the accuracy of estimating breeding values for traits with high heritability is higher than that with low heritability ${ }^{62}$. Because in traits with high heritability, phenotype of individual is closer to the genetic value and therefore the breeding value of individuals is more accurately estimated ${ }^{45}$. Heritability for berry weight has been seen in the range of 0.49 to $0.92^{15,22,51}$. In one study narrow-sense heritability was estimated 0.63 for berry weight, 0.69 for berry width, 0.68 for berry length, 0.58 for seediness, 0.48 for Brix index and 0.36 for acidity ${ }^{67}$.

\section{Conclusions}

Results of present study revealed the importance of considering population structure and relatedness factors in table grape association analysis. Seven DNA markers were found to be significantly associated with regions controlling studied pomological traits that can be useful in marker-assisted breeding programs. In addition, breeding values were estimated for Iranian table grapevine germplasm by integrating DNA maker data and pomological traits. Considering the sum of the breeding values of all the studied traits, "Taifi”, "Qzl Ouzum”, “Rishbaba Qermez", “Garmian”, “Agh Shani”, "Lal Qermez”, "Sahebi Qermez", "Saghal Solian" cultivars had the highest rank. The cultivar with high and positive breeding value can be used as a good parent in hybridization programs because they can better transfer their suitable characteristics to the progeny.

\section{Methods}

Germplasm. Forty-five Iranian table grape ( $V$. vinifera subspecies vinifera) cultivars were kindly provided from the germplasm bank of Kahriz Horticultural Research Station (Urmia, West Azarbaijan) (Table 6). 
Table 6

Characteristic of grape genotypes utilized in investigating genetic diversity using SSR

\begin{tabular}{|llllll|}
\hline Genotype & Use & Seed & Genotype & Use & Seed \\
\hline Rezghi & Table & Yes & Kalati & Table & Yes \\
\hline Hosseini & Table & Yes & Mam Braima & Table, Raisin & Yes \\
\hline Tabarze Sefid & Table & Yes & Bol Mazu & Juice & Yes \\
\hline Saghal Solian & Table & Yes & Lal Qermez & Table & Yes \\
\hline At Ouzum & Juice & Yes & Sefid Shakh Shakh & Juice & No \\
\hline Lal Seyah & Table & Yes & Alhaghi & Table & Yes \\
\hline Seyah Sardasht & Table, Raisin & Yes & Askari & Table & No \\
\hline Garmian & Table & Yes & Bidane Sefid & Table, Raisin & No \\
\hline Maiemo & Juice & Yes & Rejin & Juice & No \\
\hline Rishbaba Qermez & Table & Yes & Sarghola & Table & Yes \\
\hline Taifi & Table & Yes & Chava Ga & Juice & Yes \\
\hline Bidane Qermez & Table & No & Yaghoti & Table & Yes \\
\hline Fakhri & Table, Raisin & Yes & Qara Gandoma & Juice & Yes \\
\hline Shahroudi & Table & Yes & Gazandaii & Table & Yes \\
\hline Qara Shani & Juice & Yes & Qzl Ouzum & Table & Yes \\
\hline Sahebi Qermez & Table & Yes & Agh Shani & Table & Yes \\
\hline Inah Amjai & Table & Yes & Jig Jiga & Table & Yes \\
\hline Tabarze Qermez & Table & Yes & Lal Sefid & Table & Yes \\
\hline Dastarchin & Juice & Yes & Klkarevi & Juice & Yes \\
\hline Rishbaba Sefid & Table & Yes & Sachakh & Juice & Yes \\
\hline Agh Melhi & Juice & Yes & Shirazi & Table & Yes \\
\hline Goi Melki & Table & Yes & Angotka & Juice & Yes \\
\hline Sayani & Yes & & & \\
\hline
\end{tabular}

Phenotyping protocols. Fourteen pomological traits including total soluble solids (Brix), fruit pH, fruit titratable acidity (TA), berry weight (g), flesh weight (g), juice volume $(\mathrm{ml})$, seed weight $(\mathrm{g})$, seed number per berry, cluster width $(\mathrm{cm})$, cluster length $(\mathrm{cm})$, cluster weight (g), fruit set in open pollination $(\%)$, fruit set in controlled pollination (\%) and pollen germination rate (\%) were measured onto 12 plants per cultivar during three successive years. TSS was determined by refractometer in Brix degrees. TA was measured by the amount of $0.10 \mathrm{~N} \mathrm{NaOH}$ used to adjust the fruit juice $\mathrm{pH}$ to 8.1 . Fruit pH values were measured by $\mathrm{pH}$ meter. Berry and flesh weight, cluster weight and also single seed weight were determined by digital scale. Fruit juice of $100 \mathrm{~g}$ of each cultivar was evaluated with graduated cylinder. To measure the length and width of the cluster, three clusters from each vine were selected from the same positions and the traits were measured. To determine pollen germination, clusters were harvested in $50-70 \%$ flowering stage and cultured on medium with $1 \%$ agar and $5 \%$ sucrose $24 \mathrm{~h}$ at $26^{\circ} \mathrm{C}$. The number of germinated pollen was counted in seven microscopic areas. Percent of fruit set was calculated with the following formula: Fruit set $=$ Number of berry $/$ number of flower $\times 100$.

Marker identification and analysis. Genomic DNA was extracted by Doyle \& Doyle (1990) method ${ }^{14}$. Thirty-nine SSR primer pairs were used for preparing the molecular profile of studied cultivars (Table 3). PCR was carried out in a final volume of $20 \mu$ including 20 ng of genomic DNA, 1.75 mM magnesium chloride, $2 \mu \mathrm{l} 10 \times$ polymerase chain reaction buffer $(500 \mathrm{mM} \mathrm{KCl}, 500 \mathrm{mM}$ Tris-Hydrogen chloride; pH 8.4), $0.25 \mathrm{mM}$ of each dNTP (Cinagene Co., Tehran, Iran), $10 \mu \mathrm{M}$ of each primer and 1.1 Unit of Taq DNA polymerase (Cinagene Co., Tehran, Iran), and distilled water in a 96-well Mastercycler Gradient thermal cycler (Type 5331; Eppendorf AG, Hamburg, Germany). The PCR reaction program consisted of an initial denaturation at $94^{\circ} \mathrm{C}$ for 4 minute, followed by 35 cycles of $94^{\circ} \mathrm{C}$ for 1 minute, annealing temperature $\left(50\right.$ to $\left.59^{\circ} \mathrm{C}\right)$ for 1 minute, and $72^{\circ} \mathrm{C}$ for 2 minute, with a final extension of 7 minute at $72^{\circ} \mathrm{C}$. Amplification was checked with $3 \%(w / v)$ ultra-pure agarose gel. All PCR amplifications were scored as codominant manners.

Descriptive statistics and correlation coefficients among pomological characters were calculated in SAS 9.4 software. Genetic parameters including number of allele per SSR loci, gene diversity, heterozygosity and PIC were calculated by GenAIEx 6 software ${ }^{43}$. Cluster and discriminant analysis of principal components (DAPC) analyses for testing genetic relationships between groups were performed by using adegenet package in the $\mathrm{R}$ environment ${ }^{35}$. The find clusters function was carried out to identify the number of subpopulations. It uses K-means clustering algorithm which decomposes the total variance of a variable into within- and between-group components. The best number of subpopulations shows the lowest Bayesian Information Criterion (BIC) value. Accurate 
classification of table grape cultivars into appropriate sub-populations and detection of cultivars with mixed structure was performed using Bayesian method in Structure 2.3.4 software ${ }^{46}$. The initial value of $\mathrm{K}$ was considered in the range of 1 to 10 . For each subpopulation 10 replicates were assigned. Burn-in period length was adjusted to 100,000, followed by 100,000 Markov Chain Monte Carlo (MCMC) replications. Marker-trait association was tested by Q + K mixed model in TASSEL 2.1 software ${ }^{69}$. The linkage disequilibrium (LD) was calculated with TASSEL ${ }^{7}$.

Predicting breeding values were carried out using BLUP in SAS software ${ }^{5}$. The structure of the mixed model for estimating breeding values is as follows:

$Y=X b+Z u+e$

Where, $\mathrm{Y}$ is the vector of phenotypic values, $\mathrm{b}$ is vector of fixed effects, $\mathrm{U}$ is vector of random effects, $\mathrm{X}$ and $\mathrm{Z}$ are the design matrices, and $\mathrm{e}$ is the vector of random residual ${ }^{45}$. The breeding values estimates can be realized by solving the mixed model equations (MME) ${ }^{31}$.

$\left[\begin{array}{cc}x^{\prime} R^{-1} X & x^{\prime} R^{-1} z \\ z^{\prime} R^{-1} x & z^{\prime} R^{-1} z+G^{-1}\end{array}\right]\left[\begin{array}{l}\hat{\beta} \\ \hat{u}\end{array}\right]=\left[\begin{array}{c}x^{\prime} R^{-1} y \\ z^{\prime} R^{-1} y\end{array}\right]$

$\operatorname{BLUP}(\mathrm{U})=\left[\begin{array}{ll}\mathrm{z}^{\prime} \mathrm{x} & \mathrm{z}^{\prime} \mathrm{z}+\mathrm{Ia}\end{array}\right]^{-1}\left[\begin{array}{c}\mathrm{x}^{\prime} \mathrm{y} \\ \mathrm{z}^{\prime} \mathrm{y}\end{array}\right]=\left[\begin{array}{c}\mathrm{u}_{1} \\ \mathrm{u}_{2} \\ \vdots \\ \mathrm{u}_{\mathrm{n}}\end{array}\right]$

\section{Declarations}

\section{Author contribution statement}

M.R. contributed to the experimental activities, data collection, results explanations and writing-original draft. R.D. designed and supervised the work. H.D.B. involved in preparing the plant materials. R.D. and H.A. contributed to analyse of the data. R.D. contributed to review and edit of manuscript. All authors contributed to the discussion. The authors accept full responsibility for the context of the manuscript.

\section{Competing interests}

The authors declare that they have no conflict of interest.

\section{Permission for land study}

The authors declare that all land experiments and studies were carried out according to authorized rules.

\section{References}

1. Al-Maskri, A. H., Sajjad, M. \& Khan, S. H. Association mapping: a step forward to discovering new alleles for crop improvement. Int. J. Agric. Biol, 14, 153160 (2012).

2. Barnaud, A., Laucou, V., This, P., Lacombe, T. \& Doligez, A. Linkage disequilibrium in wild French grapevine. Vitis vinifera L subsp Silvestris. Heredity. 104, 431-437(2010).

3. Battilana, J. et al. Linkage mapping and molecular diversity at the flower sex locus in wild and cultivated grapevine reveal a prominent SSR haplotype in hermaphrodite plants. Mol. Biotechnol, 54, 1031-1037 (2013).

4. Bauer, A. M., Reetz, T. C. \& Léon, J. Estimation of breeding values of inbred lines using best linear unbiased prediction (blup) and genetic similarities. Crop. Sci, 46, 2685-2691 (2006).

5. Bernardo, R. Breeding for quantitative traits in plants 2nd edn (Stemma Press, Woodbury, M N., 2010).

6. Bouquet, A. \& Donglot, Y. Inheritance of Seedlessness in Grapevine (Vitis vinifera L.). Vitis, 35, 35-42 (1996).

7. Bradbury, P. J. et al. TASSEL: software for association mapping of complex traits in diverse samples., 23, 2633-2635 (2007).

8. Breseghello, F. \& Sorrells, M. E. Association analysis as a stategy for improvement of quantitative traits in plants. Crop Sci, 46, 1323-1330 (2006).

9. Cabezas, J. A., Cervera, M. T., Ruiz-Garcia, L., Carreño, J. \& Martínez-Zapater, J. M. A genetic analysis of seed and berry weight in grapevine. Genome, 49, 1572-1585 (2006).

10. Chen, J. et al. Construction of a high-density genetic map and QTLs mapping for sugars and acids in grape berries. BMC Plant Biol, 15, 28 (2015).

11. Chitwood, D. H. et al. A modern ampelography: a genetic basis for leaf shape and venation patterning in grape. Plant Physiol, 164, 259-272 (2014).

12. Commbe, B. G. Reserarch on development and ripening of the grape berry. Am. J. Enol. Vitic, 43, 101-110 (1992).

13. de Souza, V. A., Byrne, D. H. \& Taylor, J. F. Predicted breeding values for nine plant and fruit characteristics of 28 peach genotypes. J. Am. Soc. Hortic. Sci, $125,460-465$ (2000).

14. Doyle, J. J. \& Doyle, J. L. Isolation of plant DNA from fresh tissue. Focus, 12, 13-15 (1990).

15. Eibach, R. Investigations about the influence of some physiological and phenological characteristics on quality and their heredity. In: Proc. 5th Intern.

Symp. on Grape Breeding, St. Martin/Pfalz, 12-16 September, 1989, pp. 149-158 (1990).

16. Emanuelli, F. et al. A candidate gene association study on Muscat flavor in grapevine (Vitis vinifera L.). BMC Plant Biolo, 10, 241 (2010).

17. Falconer, D. S. \& IntroductiontoQuantitativeGenetics 2nd Edn. NewYork, NY:Longmanlnc(1981).

Page 15/19 
18. Falconer, D. S. Introduction to quantitative genetics. 3nd ed. Longman Sci. and Technol., London(1989).

19. Fanizza, G., Lamaj, F., Costantini, L., Chaabane, R. \& Grando, M. S. (2005). QTL analysis for fruit yield components in table grapevines (Vitis vinifera). Theor. Appl. Genet. 111, 658-664 (2005).

20. Fehr, W. Principles of cultivar development: Theory and technique (Macmillian Publishing Company, 1991).

21. Fernandez, L. et al. Haplotype diversity of VvTFL1A gene and association with cluster traits in grapevine (V. vinifera). BMC Plant Biol, 14, 209 (2014).

22. Firoozabady, E. \& Olmo, H. P. Heritability and correlation studies of certain quantitative traits in table grapes, Vitis ssp. Vitis, 26, 132-146 (1987).

23. Fournier-Level, A. et al. Quantitative genetic bases of anthocyanin variation in grape (Vitis vinifera L. ssp.sativa) berry: a quantitative trait locus to quantitative trait nucleotide integrated study. Genetics, 183, 1127-1139 (2009).

24. Fournier-Level, A., Hugueney, P., Verries, C., This, P. \& Ageorges, A. Genetic mechanisms underlying themethylation level of anthocyanins in grape (Vitis vinifera L). BMC Plant Biol, 11, 179 (2011).

25. Gomez, C., Alvarez, M. \& Mosquera, T. Association mapping, a method to detect quantitative trait loci: statistical bases. Agron. Colomb, 29, 367-376 (2011).

26. Grassi, F., Labra, M., Scienza, A. \& Imazio, S. Chloroplast SSR markers to assess DNA diversity in wild and cultivated grapevine. Vitis, 41, 157-158 (2002).

27. Gupta, P. K., Rustgi, S. \& Kulwal, P. L. Linkage disequilibrium and association studies in higher plants: Present status and future prospects. Plant Mol. Biol, 57, 461-485 (2005).

28. Hansche, P. E. Response to selection,p. 154-171. In J.N. Moore and J. Janick (eds.). Methods in fruit breeding.Purdue Univ. Press, West Lafayette, Ind (1983).

29. Henderson, C. R. Best linear unbiased estimation and prediction under a selection model. Biometrics, 31, $423-447$ (1977).

30. Henderson, C. R. Estimation of variances and covariances under multiple trait models. J. Dairy Sci, 67, 1581-1589 (1983).

31. Henderson, C. Statistical methods in animal improvement: Historical overview. Advances in statistical methods for genetic improvement of livestock. Springer. pp. 2-14(1990).

32. Hittalmani, S. et al. Identification of QTL for growth and grain yield-related traits in rice across nine locations of Asia. Theor. Appl. Genet, 107, 679-690 (2003)

33. Houel, C. et al. Patterns of sequence polymorphism in the fleshless berry locus in cultivated and wild Vitis vinifera accessions. BMC Plant Biol, 10, 284299 (2010).

34. Huang, Y. F. et al. Dissecting genetic architecture of grape proanthocyanidin composition through quantitative trait locus mapping. BMC Plant Biol, 12, 30-59 (2012).

35. Jombart, T., Devillard, S. \& Balloux, F. Discriminant analysis of principal components: a new method for the analysis of genetically structured populations. BMC Genetics, 11, 94 (2010).

36. Jun, T. H., Van, K., Kim, M. Y., Lee, S. H. \& Walker, D. R. Association analysis using SSR markers to find QTL for seed protein content in soybean. Euphytica, 62, 179-191 (2008).

37. Lijavetzky, D. et al. Molecular genetics of berry colour variation in table grape. Mol. Genet. Genom, 276, 427-435 (2006).

38. Liu, H. F., Wu, B. H., Fan, P. G., Li, S. H. \& Li, L. S. Sugar and acid concentrations in 98 grape cultivars analyzed by principal component analysis. J. Sci. Food Agric, 86, 1526-1536 (2006).

39. Lowe, K. M. \& Walker, M. A. Genetic linkage map of the interspecific grape rootstock cross Ramsey (Vitis champinii) $\times$ Riparia Gloire (Vitis riparia). Theor. Appl. Genet, 112, 1582-1592 (2006).

40. Meyer, K. WOMBAT-A tool for mixed model analyses in quantitative genetics by restricted maximum likelihood (REML). J. Zhejiang Univ. Sci. B, 8, 815821 (2007).

41. McGovern, P. E. (2003). Ancient Wine: the Search for the Origin of Viniculture. Princeton University Press, Princeton and Oxford, UK (2003).

42. Nelson, K. E. (1985). Harvesting and handling California table grapes for market. Bulletin 1913. 72 p. University of California Press, DANR Publications, Oakland, California, USA (1985).

43. Peakall, R. \& Smouse, P. E. GENALEX 6: genetic analysis in Excel. Population genetic software for teaching and research. Mol. Ecol. Notes, 6, 288-295 (2006).

44. Pereira, M., Ribeiro, H., Cunha, M. \& Abreu, I. Comparison of pollen quality in Vitis vinifera L. cultivars. Sci. Hortic, 227, 112-116 (2018).

45. Piepho, H. P., Mohring, J. \& Melchinger, A. BLUP for phenotypic selection in plant breeding and variety testing. Euphytica, 161, 209-228 (2008).

46. Pritchard, J. K., Stephanes, M. \& Rosenberg, N. A. Association mapping in structured populations. Am. J. Hum. Genet, 67, 170-181 (2000).

47. Riaz, S., Krivanek, A. F., Xu, K. \& Walker, M. A. Refined mapping of the Pierce's disease resistance locus, PdR1, and sex on an extended genetic map of Vitis rupestris $\times$ V. arizonica. Theor. Appl. Genet, 113, 1317-1329 (2006).

48. Roy, J. K., Bandopadhyay, R., Rustgi, S., Balyan, H. S. \& Gupta, P. K. Association analysis of agronomically important traits using SSR, SAMPL and AFLP markers in bread wheat. Curr. Sci, 90, 5-10 (2006).

49. Searle, S. R., Casella, G. \& McCulloch, C. E. Variance components (John Wiley \& Sons, Hoboken, 2009).

50. Semagn, K., Bjørnstad, A. \& Xu, Y. The genetic dissection of quantitative traits in crops. Electron. J. Biotechnol, 13, 1-45 (2010).

51. Singh, R. \& Jalikop, S. H. Studies on variability in grape. Indian J. Hortic, 43, 207-209 (1986).

52. Shiraishi, M. Three descriptors for sugars to evaluate grape germplasm. Euphytica, 71, 9-106 (1993). 
53. Spataro, G. et al. Genetic diversity and structure of a worldwide collection of Phaseolus coccineus L. Theor. Appl. Genet, 122, 1281-1291 (2011).

54. Tancred, S. J. \& Zeppa, A. G. Heritability and patterns of inheritance of the ripening date of apples. Hort Sci, 30, 325-328 (1995).

55. Tangolar, S., Eti, S., Gok, S. \& Ergenoglu, F. Obtaining plants From seedless $\times$ seedless grape crosses using embryo culture. Turk. J. Agric. For, 23, $935-942$ (1999).

56. Tello, J. et al. Polymorphisms and minihaplotypes in the VvNAC26 gene associate with berry size variation in grapevine. BMC Plant Biol, 15, 253 (2015).

57. This, P., Lacombe, T., Cadle-Davidson, M. \& Owens, C. L. Wine grape (Vitis vinifera L.) color associates with allelic variation in the domestication gene VvmybA1. Theor. Appl. Genet, 114, 723-730 (2007).

58. Tuberosa, R., Gill, B. S. \& Quarrie, S. A. Cereal genomics: Ushering in a brave new world. Plant Mol. Biol, 48, 744-755 (2002a).

59. Tuberosa, R. et al. Mapping QTLs regulating morphophysiological traits and yield in droughtstressed maize: case studies, shortcomings and perspectives. Ann. Bot, 89, 941-963 (2002b).

60. Vargas, A. M., Fajardo, C., Borrego, J., De Andrés, M. T. \& Ibanez, J. Polymorphisms in VvPel associate with variation in berry texture and bunch size in the grapevine. Aust. J. Grape Wine Res, 19, 193-207 (2013a).

61. Vargas, A., Le Cunff, L., This, P., Ibanez, J. \& Teresa, M. VvGAl1 polymorphisms associate with variation for berry traits in grapevine. Euphytica, 191, 85-95 (2013b).

62. Villumsen, T. M. \& Janss, L. Bayesian genomic selection: the effect of haplotype length and priors. BMC Proc. 3(Suppl), S11(2009).

63. White, T. L., Hodge, G. R. \& Delorenzo, M. A. Best linear prediction of breeding values in forest tree improvement, p. 99-122. In: Proc. Genet. Breeding Southern Pines. Gainesville, Fla(1986).

64. White, T. L. \& Hodge, G. R. Best linear prediction of breeding values in a forest tree improvement program. Theor. Appl. Genet, 76, 719-727 (1988).

65. White, T. L. \& Hodge, G. R. Predicting breeding values with application in forest tree improvement (Kluwer Academic Publ., Dordrecht, The Netherlands, 1989).

66. Winkler, A. J., Cook, J. A., Kliewer, W. M. \& Lider, L. A. General Viticulture 430PP (University of California Press, Berkeley and Los Angeles, 1997).

67. Xianming, W., Sykes, S. R. \& Clingeleffer, P. R. An investigation to estimate genetic parameters in CSIRO's table grape breeding program. 2. Quality characteristics. Euphytica, 128, 343-351 (2002).

68. Yu, J. \& Buckler, E. S. Genetic association mapping and genome organization of maize. Curr. Opin. Biotechnol, 17, 155-160 (2006).

69. Yu, J. et al. A unified mixed model method for association mapping that accounts for multiple levels of relatedness. Nat. Genet, 38, 203-208 (2006).

70. Zhang, Q., Wu, C., Ren, F., Li, Y. \& Zhang, C. Association analysis of important agronomical traits of maize inbred lines with SSRs. Aust. J. Crop Sci, 6, 1131-1138 (2012).

\section{Figures}



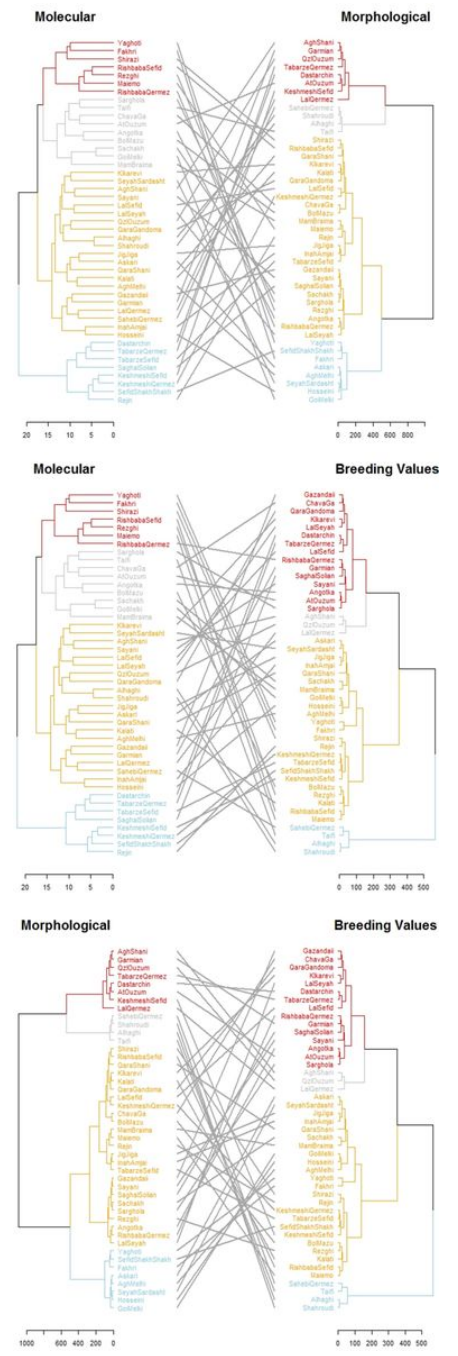

\section{Figure 1}

Synteny analysis of distribution of genotypes across dendrograms including comparison of morphological and molecular data hierarchical cluster dendrograms, comparison of morphological and breeding value hierarchical cluster dendrograms and comparison of molecular data and breeding value hierarchical cluster dendrograms

(a)

(c)
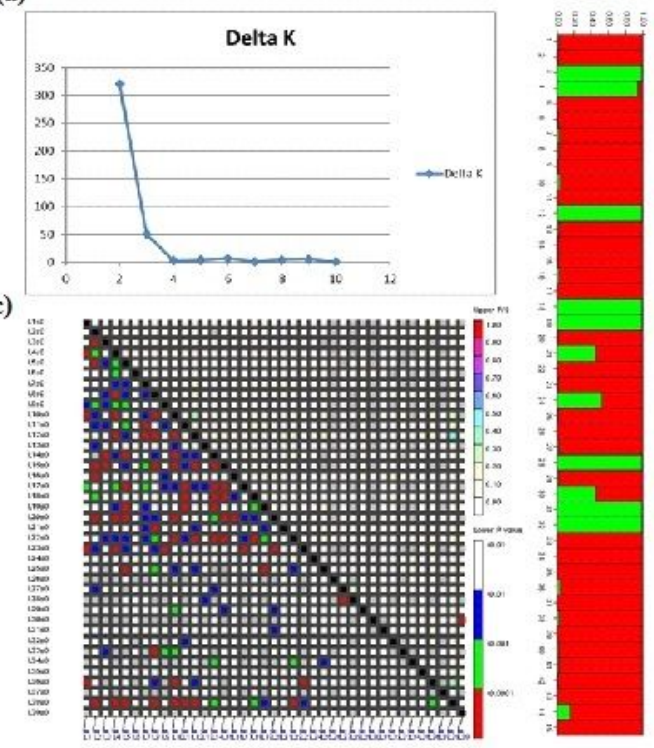

(b)

Figure 2 
(a) Bilateral charts to determine the optimal number of $\mathrm{K}$ identified by Structure program. (b) Population structure indicating the two groups identified with different color bars. (c) LD plot generated by retrotransposon marker pairs in 45 Iranian grape (V. vinifera subspecies vinifera) cultivars. The upper diagonal shows $\mathrm{r} 2$ among each pair of markers. The lower diagonal shows the levels of significance between each pair of markers

(a)

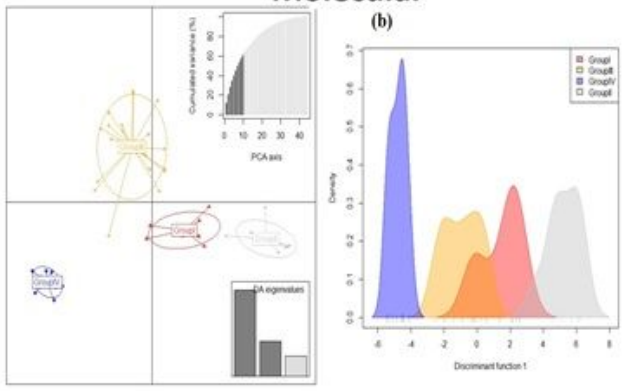

(a)

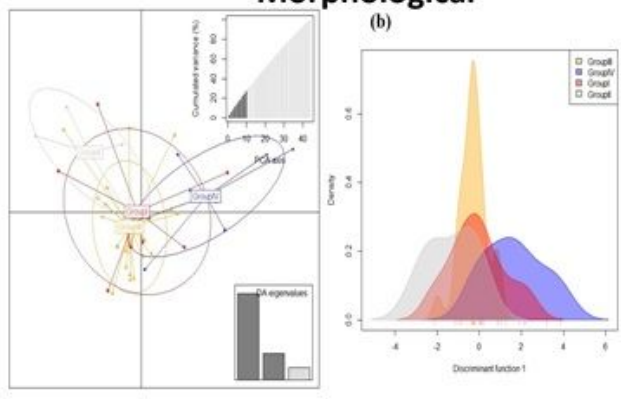

(a)

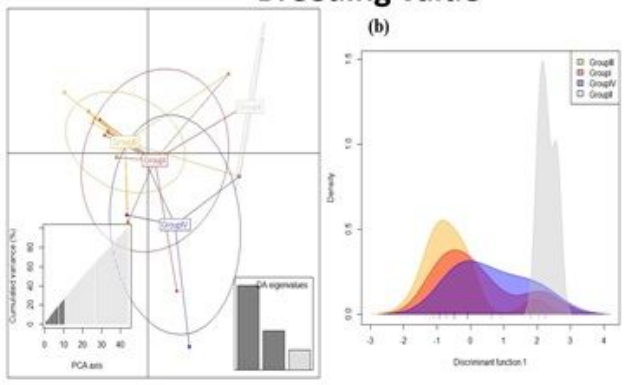

Figure 3

Discriminant analysis of principal components (DAPC) between groups resulting from molecular, morphological and breeding value cluster analysis. (a) Dots represent individuals with colors denoting cluster allocation. Percentages of cumulated variance explained by principal component 1 (PC1) to PC10 are shown in the top right corner. (b) Density plot of individuals along the first discriminant function from the discriminant analysis of principal components (DAPC) for groups 1, 2, 3 and 4 